Li, Y., X. Shi, A. Emrouznejad and L. Liang (2018) Ranking intervals for two-stage production systems,

Journal of Operational Research Society, https://doi.org/10.1080/01605682.2018.1535267

\title{
Ranking intervals for two-stage production systems
}

\author{
Yongjun Li, Xiao Shi *, Ali Emrouznejad and Liang Liang
}

\begin{abstract}
Traditional Data Envelopment Analysis (DEA) models find the most desirable weights for each Decision Making Unit (DMU) in order to estimate the highest efficiency score as possible. Usually, decision makers are using these efficiency scores for ranking the DMUs. The main drawback in this process is that the ranking based on weights obtained from the standrad DEA models ignore other feasible weights, this is due to the fact that DEA may have multiple solutions for each DMU. To overcome this problem, Salo and Punkka (2011) deemed each DMU as a "Black box" and developed a mix-integer model to obtain the ranking intervals for each DMU over sets of all its feasible weights.

In many real world applications, there are DMUs that have a two-stage production system. In this paper, we extend the Salo and Punkka (2011)'s model to more common and practical applications considering the two-stage production structure. The proposed approach calculates each DMU's ranking interval for the overall system as well as ranking interval for each subsystem/sub-stage. An example is given to illustrate the applicability of the proposed approach while an application for non-life insurance companies has been discussed to show the usefulness of this method. A real application in Chinese commercial banks shows how this approach can be used by policy makers.
\end{abstract}

Keywords: Ranking intervals, Data Envelopment Analysis, two-stage production systems

\section{Introduction}

Data Envelopment Analysis (DEA), first developed by Charnes et al. (1978), has been proven as an effective tool for performance evaluation and benchmarking. This technique makes no assumptions on the production function and imposes no subjective weights on multiple inputs and multiple outputs. DEA has been widely applied in many 
areas (Emrouznejad and Yang, 2017). The DEA technique allows a DMU to choose the most favorable weights to achieve the best possible relative efficiency. Then, these efficiency scores serve as a basis for obtaining the rankings of the DMUs. But when there are multiple decision making units, the traditional DEA model present difficulty in ranking the decision-making units as there may be two or more efficient DMUs. Thus, it makes raking DMUs unavailable. In order to overcome this issue, Andersen and Petersen (1993) proposed a super-efficiency data envelopment analysis (SEDEA) and the model could be used in ranking the performance of efficient DMUs. Afterwards, super-efficiency DEA has been extended and applied to many areas (e.g. Zhu, 2001;Ray, 2008; Sadjadia et al. 2011; Du et al. 2014; Banker et al. 2017). However, the standard ranking procedure does not consider all the possible weights as it only considers the weights most favorable to each DMU. The main issue that has been ignored in the past literature is that, the rankings of a DMU relative to other DMUs can change over different weights when applying the DEA models. Hence, it is important to consider all possible weights to evaluate each DMU.

To overcome this problem, Salo and Punkka 2011 have proposed a procedure to rank DMUs by taking into account all possible weights (see also Yang et al. 2012). They have introduced an interval for all possible rankings that is determined by the best and worst ranking. For each DMU, the best ranking is defined as the minimum number of other DMUs with strictly larger efficiency scores, while the worst ranking is defined on the maximum number of other DMUs with larger or equal efficiency scores. For this purpose, Salo and Punkka (2011) developed mix-integer models to obtain the ranking interval for each DMU over sets of all feasible weights. Alcaraz et al. (2013) applied similar mix-integer models in cross-efficiency evaluation to obtain the ranking interval for each DMU. Besides, Yang et al. (2012) obtained the best and worst ranking of each DMU over all possible weights by using an acceptability analysis constructed from the resulting matrix of interval cross-efficiencies. All these methods for obtaining the ranking intervals treated each DMU as a "Black Box". Thus, they ignored the internal structure of the production system.

However, as discussed in many DEA studies, in many real applications, DMUs 
have a two-stage structure. The issue of two-stage DEA has been extensively studied by Seiford and Zhu (1999), Chen and Zhu (2004), Kao and Hwang (2008), Chen et al. (2009) $)^{\mathrm{a}}$, Chen et al. (2009) ${ }^{\mathrm{b}}$, Fukuyama and Weber (2010), Zha and Liang (2010) and Li et al. (2012). Seiford and Zhu (1999) proposed an standard two stage DEA model. Kao and Hwang (2008) considered two sub-stage production where the immediate products are considered as the outputs of sub-stage 1 and the inputs to the sub-stage 2 . Zha and Liang (2010) and Li et al. (2012) extended two-stage DEA models by considering freely distributed inputs of two subsystems and the additional inputs to the second stage, respectively. Chen and Zhu (2004) and Kao and Hwang (2008) developed efficiency measurement framework for classic two-stage systems. The first subsystem uses inputs to produce outputs, which then become the inputs to the subsequent subsystem. The second subsystem thus consumes these outputs from the first subsystem to produce its outputs. Zha and Liang (2010) expanded the technology sets of each subsystem by considering the shared inputs between two serial subsystems. More recently, Li et al. (2012) further extended the two-stage network structures by assuming there existed exogenous inputs to the second subsystem. As results, two-stage DEA has been extensively applied to many areas, such as hotels (Sexton and Lewis, 2003; Huang et al. 2014), banks (Wang et al. 2014, Shi et al. 2017), insurance companies (Kao and Hwang, 2008; Eling and Schaper, 2017), industry (Wu et al. 2017; Zhao et al. 2017), airports (Lozano et al. 2013; Tsui et al. 2014) and so on.

In this paper, we develop a method to obtain the ranking intervals for the classic two-stage production systems as discussed by Seiford and Zhu (1999), Chen and Zhu (2004), and Kao and Hwang (2008). That is, the first subsystem uses inputs to produce outputs that then become the inputs to the second subsystem to produce the final outputs. The proposed model calculates each DMU's ranking intervals for the overall system as well as two subsystems. We belive that this process provides more accurate information for decision makers by identifying the best (and/or worst) DMUs in the overall system and both subsystems over all feasible weights. Besides, the proposed approach provides information regarding the sensitivity of the DMU's ranking intervals for the overall system and both two subsystems over sets of all feasible weights. 
The remainder of this paper is organized as follows. In the next section, the ranking interval procedure of Salo and Punkka (2011) has been reviewed briefly. Then, in Section 3, a method is developed to obtain the ranking interval considering the twostage production systems. This is followed by illustration example in Section 4. An application is also given in this section to show the usefulness of the proposed procedure. In Section 5, the method is extended to measure the ranking interval for more general two-stage systems. Finally, conclusions and direction for future research are given in Section 6.

\section{Ranking intervals by Salo and Punkka (2011)}

Assume that there are $n$ DMUs denoted as $\operatorname{DMU}_{j}(j=1,2, \cdots, n)$. Each DMU uses inputs $x_{i j}(i=1, \ldots, m)$ to produce outputs $y_{r j}(r=1, \ldots, s)$. Based on the definition of Charnes et al. (1978) and Dyson et al. (2001), the efficiency of $D M U_{k}$ is defined as:

$$
E_{k}(u, v)=\frac{\sum_{r} u_{r} y_{r k}}{\sum_{i} v_{i} x_{i k}}
$$

Where $u_{r}(r=1, \ldots, s)$ and $v_{i}(i=1, \ldots, m)$ are the output weights and input weights, respectively.

For any feasible weights $u_{r}(r=1, \ldots, s)$ and $v_{i}(i=1, \ldots, m)$, Salo and Punkka (2011) defined two ranking sets based on the efficiency scores from (1):

$$
\begin{gathered}
R_{k}^{>}(u, v)=\left\{l \in\{1, \ldots, n\} \mid E_{l}(u, v)>E_{k}(u, v)\right\} \\
R_{k}^{\geq}(u, v)=\left\{l \in\{1, \ldots, n\} \backslash\{k\} \mid E_{l}(u, v) \geq E_{k}(u, v)\right\}
\end{gathered}
$$

$R_{k}^{>}$contains the indexes of those other DMUs with strictly larger efficiency scores than that of $D M U_{k}$. And $R_{k}^{\geq}$contains the indexes of those other DMUs with no less efficiency scores than that of $D M U_{k}$. That is, $R_{k}^{>}$and $R_{k}^{\geq}$contain the indexes of DMUs dominating $D M U_{k}$. The corresponding efficiency rankings are defined as $r_{k}^{>}(u, v)=1+\left|R_{k}^{>}(u, v)\right|$ and $r_{k}^{\geq}(u, v)=1+\left|R_{k}^{\geq}(u, v)\right|$, where $|R|$ denotes the cardinality of the 
set $R$.

The ranking interval of $D M U_{k}$ is then defined as $\left[r_{k}^{\min }, r_{k}^{\max }\right]$, where the best and worst rankings for $D M U_{k}$ are given by $r_{k}^{\min }=\min _{u, v} r_{k}^{>}(u, v)$ and $r_{k}^{\max }=\max _{u, v} r_{k}^{\geq}(u, v)$, respectively.

The following proposition shows how to find the best ranking $r_{k}^{\min }$ for $D M U_{k}$.

$$
\begin{aligned}
r_{k}^{\min }= & \min _{u, v} \quad r_{k}^{>}(u, v) \\
= & \min _{u, v, p} 1+\sum_{l \neq k} p_{l} \\
\text { s.t. } \quad & \sum_{r} u_{r} y_{r l} \leq \sum_{i} v_{i} x_{i l}+C p_{l} \quad l=1, \ldots, n,(l \neq k) \\
& \sum_{r} u_{r} y_{r k}=\sum_{i} v_{i} x_{i k}=1 \\
& p_{l} \in\{0,1\}, l \neq k \\
& u_{r}, v_{i} \geq 0, r=1, \ldots, s ; i=1, \ldots, m
\end{aligned}
$$

where $\mathrm{C}$ is a large positive constant. Model (2) is a mixed-integer linear problem. The constraints (2.1) and (2.2) assure that $D M U_{l}$ has larger efficiency score than that of $D M U_{k}$. The optimal value of model (2) is the best ranking of $D M U_{k}$ over sets of all feasible weights. The best ranking of $D M U_{k}$ is defined as the minimum number of other DMUs that have larger efficiency scores than it. Obviously, no matter how the weights change, other DMUs dominating $D M U_{k}$ have better rankings than it.

By definition 1, the most unfavorable scenario for $D M U_{k}$ is that in which we have the minimum number of DMUs that perform worse or, equivalently, the maximum number of DMUs that perform no worse than $D M U_{k}$. The following definition establishes what we mean by the worst ranking $\left(r_{k}^{\max }\right)$ of a given $D M U_{k}$ : 


$$
\begin{aligned}
r_{k}^{\max }= & \max _{u, v} \quad r_{k}^{\geq}(u, v) \\
= & \max _{u, v, p} 1+\sum_{l \neq k} p_{l} \\
\text { s.t. } \quad & \sum_{i} v_{i} x_{i l} \leq \sum_{r} u_{r} y_{r l}+C\left(1-p_{l}\right) \quad l=1, \ldots, n,(l \neq k) \\
& \sum_{r} u_{r} y_{r k}=\sum_{i} v_{i} x_{i k}=1 \\
& p_{l} \in\{0,1\}, l \neq k \\
& u_{r}, v_{i} \geq 0, r=1, \ldots, s ; i=1, \ldots, m
\end{aligned}
$$

where $\mathrm{C}$ is a large positive constant. Model (3) is a mixed-integer linear problem. The optimal value of model (3) is the worst ranking of $D M U_{k}$ over sets of all feasible weights. The worst ranking of $D M U_{k}$ is defined as the maximum number of DMUs that have no less efficiency scores than it. Obviously, no matter how the weights change, other DMUs dominating $D M U_{k}$ have no worse rankings than it. Based on model (2) and (3), the ranking interval $\left[r_{k}^{\min }, r_{k}^{\max }\right]$ of $D M U_{k}$ can be computed. These models treat the production systems as "Black Box".

The approach can be generalized to systems composed of two subsystems connected in series. In the next section, we will discuss how to calculate the best ranking and the worst ranking for each DMU with a two-stage production system.

\section{Ranking intervals for two-stage production systems}

Suppose the operation of a DMU can be divided into two subsystems or processes, as depicted in Fig.1. For $D M U_{k}$, subsystem 1 applies inputs $x_{i k}(i=1, \ldots, m)$ to produce the intermediate products $z_{d k}(d=1, \ldots, D)$. All these intermediate products are then used by subsystem 2 to produce the final outputs $y_{r k}(r=1, \ldots, s)$. Based on the definition of Kao and Huang (2008), $D M U_{k}$ 's efficiency scores for the overall system and two subsystems are defined as:

$$
E_{k}=\frac{\sum_{r} u_{r} y_{r k}}{\sum_{i} v_{i} x_{i k}}
$$




$$
\begin{aligned}
E_{k}^{1} & =\frac{\sum_{d} w_{d}^{1} z_{d k}}{\sum_{i} v_{i} x_{i k}} \\
E_{k}^{2} & =\frac{\sum_{r} u_{r} y_{r k}}{\sum_{d} w_{d}^{2} z_{d k}}(6)
\end{aligned}
$$

where $u_{r}(r=1, \ldots, s)$ and $v_{i}(i=1, \ldots, m)$ are the output weights and input weights, respectively. Accordingly, $w_{d}^{1}(d=1, \ldots, D)$ and $w_{d}^{2}(d=1, \ldots, D)$ are the weights attached to the intermediate measures for subsystem 1 and subsystem 2, respectively. Similar to Kao and Hwang (2008) and Liang et al. (2008), we assume that the weights attached to the intermediate outputs in both subsystem 1 and subsystem 2 are the same, i.e. $w_{d}^{1}=w_{d}^{2}$. This assumption represents the serial relationship between the two subsystems (Chen et al., 2009 $)$. If we solve the two-stage DEA without this assumption, then our method is identical to independently employing the model for each subsystem. Therefore, this paper assumes $w_{d}^{1}=w_{d}^{2}=w_{d}$.

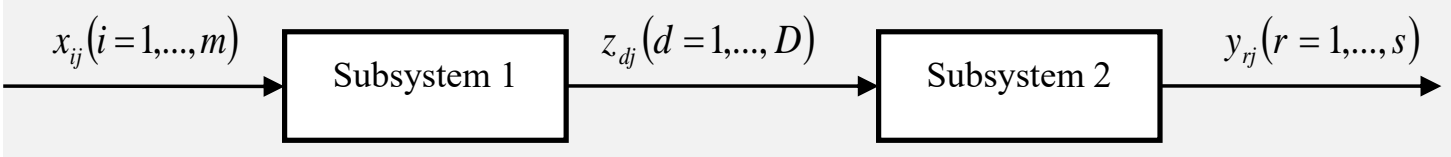

Fig. 1 Two-stage production system

\subsection{Ranking interval of a DMU for the overall system}

As discussed in section 2, choosing different weights may lead to different rankings for a "Black-Box" DMU. Similarly, choosing different weights may result in different rankings for a DMU with a two-stage production system. To obtain the ranking intervals for DMUs with a two-stage production system, we firstly give some definitions:

Definition 1. For every set of $\left(u_{r}, w_{d}, v_{i}\right), \quad r=1, \ldots s, d=1, \ldots D, i=1, \ldots, m$,

$$
R_{k}^{>}(u, w, v)=\left\{l \in\{1, \ldots, n\}\left\{\begin{array}{l}
E_{l}^{1}(u, w, v)>E_{k}^{1}(u, w, v) \text { and } E_{l}^{2}(u, w, v)>E_{k}^{2}(u, w, v) \text { or } \\
E_{l}^{1}(u, w, v) \geq E_{k}^{1}(u, w, v) \text { and } E_{l}^{2}(u, w, v)>E_{k}^{2}(u, w, v) o r \\
E_{l}^{1}(u, w, v)>E_{k}^{1}(u, w, v) \text { and } E_{l}^{2}(u, w, v) \geq E_{k}^{2}(u, w, v)
\end{array}\right\}\right.
$$


$R_{k}^{>}(u, w, v)$ contains the indexes of those other DMUs with larger efficiency scores for both subsystems, or with larger efficiency scores for one sub-system and no less efficiency scores for the other sub system than that of $D M U_{k}$. According to Kao and Hwang (2008), the overall efficiency score is the product of the efficiency scores of two sub-systems. Thus, if $D M U_{1}(l=1, \ldots, n, l \neq k)$ has larger efficiency scores for both subsystems, or has larger efficiency scores for one sub-system and no less efficiency scores for the other sub-system than that of $D M U_{k}$, then the overall efficiency score of $D M U_{l}$ would be surely larger than that of $D M U_{k}$. In such case, not only $D M U_{l}$ 's two sub-systems performed no worse than that of $D M U_{k}$, but also $D M U_{l}$ 's overall system performed better than that of $D M U_{k}$. $D M U_{1}(l=1, \ldots, n, l \neq k)$ dominates $D M U_{k}$. Therefore, $R_{k}^{>}(u, w, v)$ contains the indexes of $D M U_{1}(l=1, \ldots, n, l \neq k)$ that dominate $D M U_{k}$.

Definition 2. For every set of $\left(u_{r}, w_{d}, v_{i}\right), \quad r=1, \ldots s, d=1, \ldots D, i=1, \ldots, m$,

$$
\begin{aligned}
& R_{k}^{\geq}(u, w, v)=\left\{l \in\{1, \ldots, n\} \backslash k \mid E_{l}^{1}(u, w, v) \geq E_{k}^{1}(u, w, v) \text { and } E_{l}^{2}(u, w, v) \geq E_{k}^{2}(u, w, v)\right\} \\
& R_{k}^{\geq}(u, w, v) \text { contains the indexes of those other DMUs with no less efficiency }
\end{aligned}
$$
scores for both subsystems than that of $D M U_{k}$. The corresponding efficiency rankings for $D M U_{k}$ are defined as $r_{k}^{>}(u, w, v)=1+\left|R_{k}^{>}(u, w, v)\right|$ and $r_{k}^{\geq}(u, w, v)=1+\left|R_{k}^{\geq}(u, w, v)\right|$, respectively. If $D M U_{k}$ is CCR efficient, then its efficiency score is no less than that of other DMUs. Thus, its best ranking in Proposition 1 will be one.

Proposition 1. The optimum of the minimization problem 


$$
\begin{array}{ll}
\min _{u, w, v, p^{1}, p^{2}} & p_{l}^{1}+p_{l}^{2} \\
\text { s.t. } & \sum_{r} u_{r} y_{r l} \leq \sum_{d} w_{d} z_{d l}+C p_{l}^{2} \quad l=1, \ldots, n,(l \neq k)(7 ., \quad l=1, \ldots, n,(l \neq k) \\
& \sum_{d} w_{d} z_{d l} \leq \sum_{i} v_{i} x_{i l}+C p_{l}^{1} \quad l=1 \\
& \sum_{r} u_{r} y_{r k}=\sum_{d} w_{d} z_{d k}=1 \\
& \sum_{d} w_{d} z_{d k}=\sum_{i} v_{i} x_{i k}=1 \\
& p_{l}^{1}, p_{l}^{2} \in\{0,1\}, l \neq k \\
& u_{r}, w_{d}, v_{i}, r=1, \ldots, s ; d=1, \ldots, D ; i=1, \ldots, m
\end{array}
$$

is to identify the DMUs with strictly larger efficiency scores for both subsystems than that of $D M U_{k}$. The constraints (7.1), (7.2), (7.3) and (7.4) assure that $D M U_{l}$ has larger efficiency scores for both subsystems than that of $D M U_{k}$. If $E_{l}^{1}>E_{k}^{1} \&$ $E_{l}^{2}>E_{k}^{2}$, then the optimal value in the objective function is 2 , i.e., $p_{l}^{1^{*}}+p_{l}^{2^{*}}=2$. If $E_{l}^{1}>E_{k}^{1} \quad \& \quad E_{l}^{2} \leq E_{k}^{2}$, or if $E_{l}^{1} \leq E_{k}^{1} \quad \& \quad E_{l}^{2}>E_{k}^{2}$, then $p_{l}^{1^{*}}+p_{l}^{2^{*}}=1$ holds; if $E_{l}^{1} \leq E_{k}^{1} \quad \& \quad E_{l}^{2} \leq E_{k}^{2}$, then $p_{l}^{1^{*}}+p_{l}^{2^{*}}=0$ holds. Thus, the model has a feasible solution. The proof of this proposition is given in the Appendix.

Therefore, the optimal value in the objective function may be three values: 0,1 or 2. (1) If $p_{l}^{1^{*}}+p_{l}^{2^{*}}=2$ holds in model (7), then $D M U_{l}$ dominates $D M U_{k}$.(2) If $p_{l}^{1^{*}}+p_{l}^{2^{*}}=0$ holds, then the efficiency scores of $D M U_{l}$ and $D M U_{k}$ may satisfy one of the following conditions: $E_{l}^{1}<E_{k}^{1}$ and $E_{l}^{2}<E_{k}^{2} ; E_{l}^{1}<E_{k}^{1}$ and $E_{l}^{2}=E_{k}^{2}$; $E_{l}^{1}=E_{k}^{1}$ and $E_{l}^{2}<E_{k}^{2} ; E_{l}^{1}=E_{k}^{1}$ and $E_{l}^{2}=E_{k}^{2}$. So, $D M U_{l}$ does not dominate $D M U_{k}$ as $D M U_{l} \prec D M U_{k}$ or $D M U_{l} \approx D M U_{k}$. (3) If $p_{l}^{1^{*}}+p_{l}^{2^{*}}=1$ holds, the efficiency scores of $D M U_{l}$ and $D M U_{k}$ may satisfy one of the following conditions: $E_{l}^{1}>E_{k}^{1}$ and $E_{l}^{2}=E_{k}^{2} ; E_{l}^{1}>E_{k}^{1}$ and $E_{l}^{2}<E_{k}^{2} ; E_{l}^{1}=E_{k}^{1} \quad$ and $E_{l}^{2}>E_{k}^{2} ; E_{l}^{1}<E_{k}^{1}$ and $E_{l}^{2}>E_{k}^{2}$. Thus, if $p_{l}^{1^{*}}+p_{l}^{2^{*}}=1$ holds, then $D M U_{l}$ dominates $D M U_{k}$ $\left(D M U_{l} \succ D M U_{k}\right)$ or no dominating relationship may exist between $D M U_{l}$ and $D M U_{k}$. Therefore, by applying model (7), we could identify the DMUs dominating 
$D M U_{k}$ if $p_{l}^{1^{*}}+p_{l}^{2^{*}}=2$ holds. However, we could not identify whether $D M U_{l}$ dominates $D M U_{k}$ or no dominating relationship between them if $p_{l}^{1^{*}}+p_{l}^{2^{*}}=1$ holds. To further identify whether $D M U_{l}$ dominates $D M U_{k}$ if $p_{l}^{1^{*}}+p_{l}^{2^{*}}=1$ holds, we should identify whether $D M U_{l}$ satisfies $E_{l}^{1}>E_{k}^{1}$ and $E_{l}^{2}=E_{k}^{2}$ (or $E_{l}^{1}=E_{k}^{1}$ and $\left.E_{l}^{2}>E_{k}^{2}\right)$

If $p_{l}^{1^{*}}+p_{l}^{2^{*}}=1$ holds and $E_{l}^{1}>E_{k}^{1}$ and $E_{l}^{2} \leq E_{k}^{2}$ (From model (7)), then the following model could further identify whether $D M U_{l}$ satisties $E_{l}^{1}>E_{k}^{1}$ and $E_{l}^{2}=E_{k}^{2}$

$$
\begin{array}{ll}
\min _{u, w, v} & C \\
\text { s.t. } & \sum_{r} u_{r} y_{r l} \geq \sum_{d} w_{d} z_{d l} \quad l=1, \ldots, n,(l \neq k) \\
& \sum_{d} w_{d} z_{d l}>\sum_{i} v_{i} x_{i l} \quad l=1, \ldots, n,(l \neq k) \\
& \sum_{r} u_{r} y_{r k}=\sum_{d} w_{d} z_{d k}=1 \\
& \sum_{d} w_{d} z_{d k}=\sum_{i} v_{i} x_{i k}=1 \\
& p_{l}^{1}, p_{l}^{2} \in\{0,1\}, l \neq k \\
& u_{r}, w_{d}, v_{i}, r=1, \ldots, s ; d=1, \ldots, D ; i=1, \ldots, m
\end{array}
$$

The objective value of $C$ is constant. $D M U_{k}$ is the evaluated DMU. The constraints (8.1), (8.2), (8.3) and (8.4) assure that $D M U_{l}$ has larger efficiency score for subsystem 1 and no less efficiency score for subsystem 2 than that of $D M U_{k}$. If model (8) has a feasible solution, then $E_{l}^{1}>E_{k}^{1}$ and $E_{l}^{2}=E_{k}^{2}$. In such case, $D M U_{l}$ dominates $D M U_{k}$. If model (8) has no feasible solution, then $E_{l}^{1}>E_{k}^{1}$ and $E_{l}^{2}<E_{k}^{2}$. In such case, there exists no dominating relation between $D M U_{l}$ and $D M U_{k}$. Thus, this model could identify the DMUs dominating $D M U_{k}$. Similarly, if $p_{l}^{1^{*}}+p_{l}^{2^{*}}=1$ holds and $E_{l}^{1} \leq E_{k}^{1}$ and $E_{l}^{2}>E_{k}^{2}$ (From model (7)), then the following model could identify whether $D M U_{l}$ satisfies $E_{l}^{1}=E_{k}^{1}$ and $E_{l}^{2}>E_{k}^{2}$. 


$$
\begin{array}{ll}
\min _{u, w, v, p} & C \\
\text { s.t. } & \sum_{r} u_{r} y_{r l}>\sum_{d} w_{d} z_{d l} \quad l=1, \ldots, n,(l \neq k) \\
& \sum_{d} w_{d} z_{d l} \geq \sum_{i} v_{i} x_{i l} \quad l=1, \ldots, n,(l \neq k) \\
& \sum_{r} u_{r} y_{r k}=\sum_{d} w_{d} z_{d k}=1 \\
& \sum_{d} w_{d} z_{d k}=\sum_{i} v_{i} x_{i k}=1 \\
& p_{l}^{1}, p_{l}^{2} \in\{0,1\}, l \neq k \\
& u_{r}, w_{d}, v_{i}, r=1, \ldots, s ; d=1, \ldots, D ; i=1, \ldots, m
\end{array}
$$

The objective value of $C$ is constant. The constraints (9.1), (9.2), (9.3) and (9.4) assure that $D M U_{l}$ has no less efficiency score for subsystem 1 and larger efficiency score for subsystem 2 than that of $D M U_{k}$. If model (9) has a feasible solution, then $E_{l}^{1}=E_{k}^{1}$ and $E_{l}^{2}>E_{k}^{2}$. In such case, $D M U_{l}$ dominates $D M U_{k}$. If model (9) has no feasible solution, then $E_{l}^{1}<E_{k}^{1}$ and $E_{l}^{2}>E_{k}^{2}$. In such case, there exists no dominating relation between $D M U_{l}$ and $D M U_{k}$.

The most unfavorable scenario for $D M U_{k}$ is that in which we have the minimum number of DMUs whose two subsystems both perform worse or, equivalently, the maximum number of DMUs whose two subsystems perform no worse than $D M U_{k}$ by definition 2 . The following proposition establishes what we mean by the worst ranking of a given $D M U_{k}$.

Proposition 2. In the following model, the optimum of the maximization problem, i.e. $r_{k}^{\max }$, is the worst efficiency ranking of $D M U_{k}$ considering each DMU has a twostage production system. 


$$
\begin{aligned}
r_{k}^{\max }= & \max _{u, w, v} \mathrm{r}_{k}^{\geq}(u, w, v)= \\
= & \max _{u, w, v, p^{1}, p^{2}} 1+\sum_{l \neq k} p_{l}^{1} * p_{l}^{2} \\
\text { s.t. } \quad & \sum_{d} w_{d} z_{d l} \leq \sum_{r} u_{r} y_{r l}+C\left(1-p_{l}^{2}\right) \quad l=1, \ldots, n,(l \neq k) \\
& \sum_{i} v_{i} x_{i l} \leq \sum_{d} w_{d} z_{d l}+C\left(1-p_{l}^{1}\right) \quad l=1, \ldots, n,(l \neq k) \\
& \sum_{r} u_{r} y_{r k}=\sum_{d} w_{d} z_{d k}=1 \\
& \sum_{d} w_{d} z_{d k}=\sum_{i} v_{i} x_{i k}=1 \\
& p_{l}^{1}, p_{l}^{2} \in\{0,1\}, l \neq k \\
& u_{r}, w_{d}, v_{i}, r=1, \ldots, s ; d=1, \ldots, D ; i=1, \ldots, m
\end{aligned}
$$

The constraints (10.1), (10.2), (10.3) and (10.4) assure that $D M U_{l}$ has no less efficiency scores for both two subsystems than that of $D M U_{k}$. The optimal value $\sum_{l \neq k} p_{l}^{1^{*} *} p_{l}^{2^{*}}$ in the objective function denotes the number of DMUs with no less efficiency scores for both subsystems than that of $D M U_{k}$. When the efficiency scores

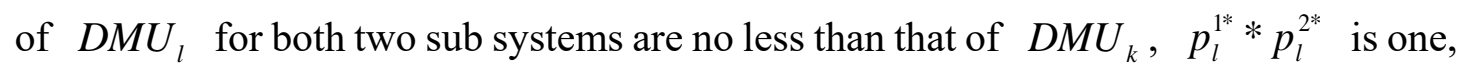
otherwise, $p_{l}^{1^{*} * p_{l}^{2^{*}}}$ is zero. The model is a mixed-integer program and non-linear. We solve it by using YALMIP toolbox in Matlab. The proof of this proposition is given in the Appendix.

The entire proposed procedure of calculating the best ranking of the whole system is depicted in a flowchart produced in Fig.2.

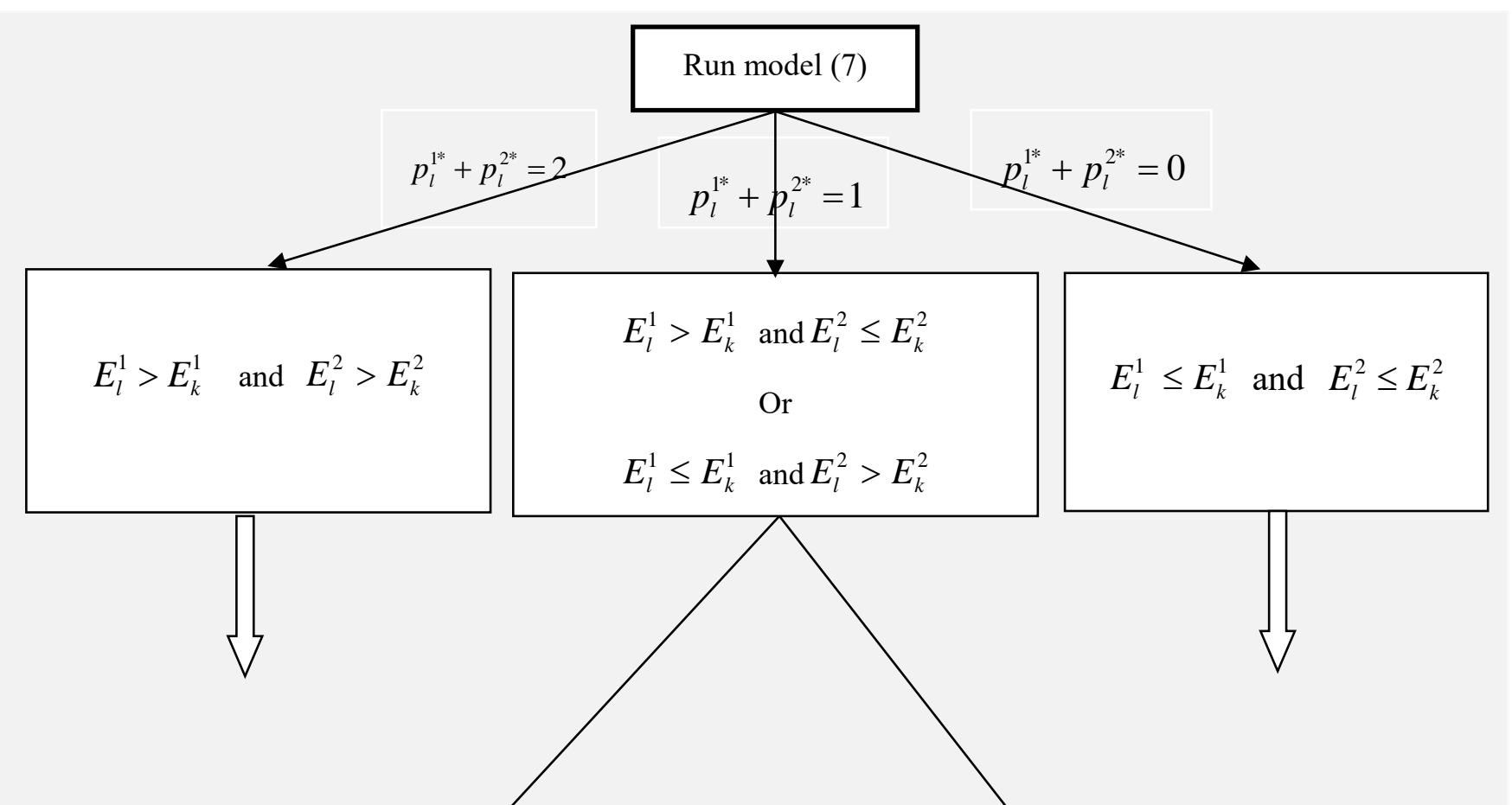


$\mathrm{DMU}_{1}$ dominates

$\mathrm{DMU}_{\mathrm{k}}$
$\mathrm{DMU}_{1}$ does not

dominates $\mathrm{DMU}_{\mathrm{k}}$
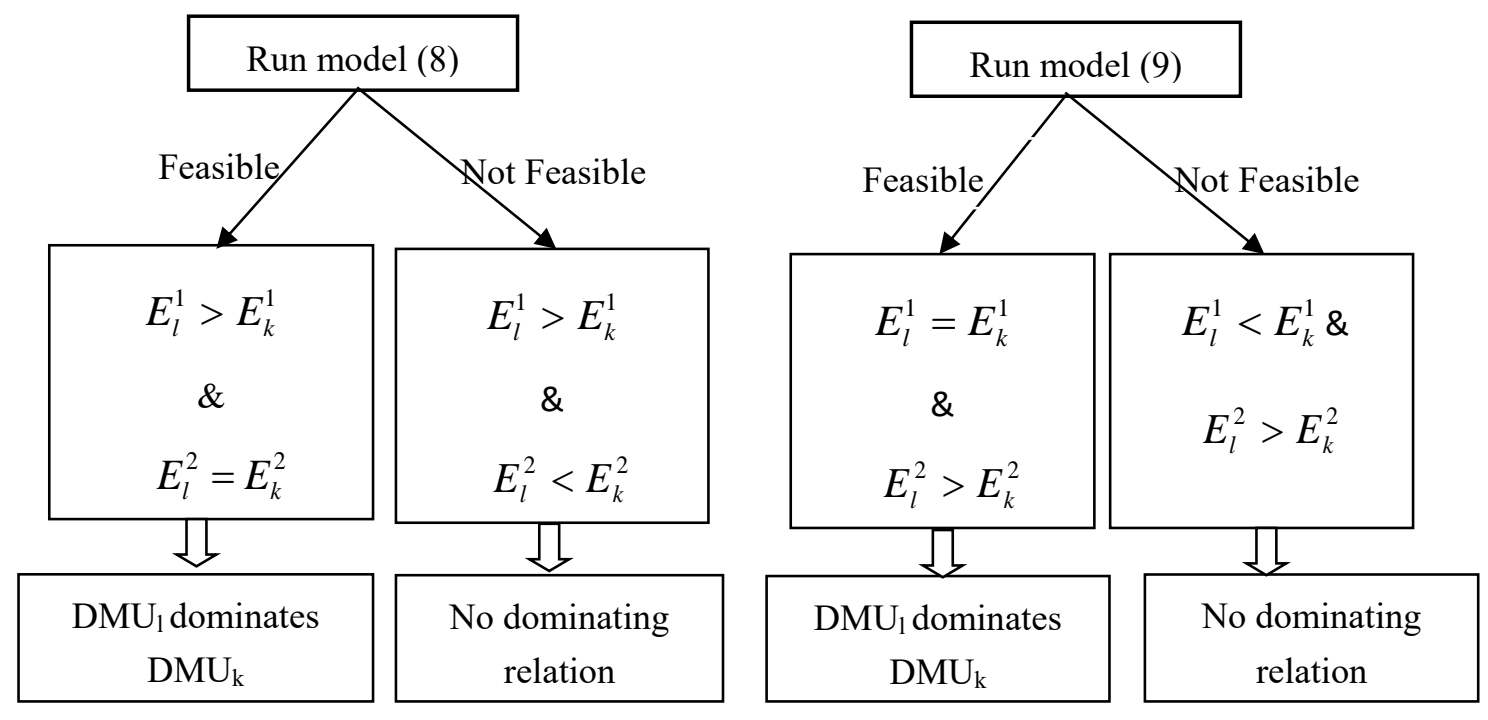

Fig.2 The flowchart of calculating the best ranking of the whole system

The proposed algorithm is summarized as follows:

First, we use model (7) to identify the possible dominating relationship between $D M U_{l} l \in\{1, \ldots, n\}$ and the evaluated $D M U_{k}$. Thus, if $p_{l}^{1^{*}}+p_{l}^{2^{*}}=2$, then $D M U_{l} \succ D M U_{k}$; if $p_{l}^{1^{*}}+p_{l}^{2^{*}}=1$, dominating relationship is unknown as $D M U_{l} \succ D M U_{k}$ or no dominating relationship exists between them; if $p_{l}^{1^{*}}+p_{l}^{2^{*}}=0$, then $D M U_{l} \prec D M U_{k}$ or $D M U_{l} \approx D M U_{k}$.

Second, we run model (8) if $p_{l}^{1^{*}}+p_{l}^{2^{*}}=1$ and $E_{l}^{1}>E_{k}^{1}$ and $E_{l}^{2} \leq E_{k}^{2}$ (from model (7)). If model (8) has feasible solution, $D M U_{l} \succ D M U_{k}$, otherwise, no dominating relationship exists between them. Similiarly, we run model (9) if $p_{l}^{1^{*}}+p_{l}^{2^{*}}=1$ and $E_{l}^{1} \leq E_{k}^{1}$ and $E_{l}^{2}>E_{k}^{2}$ (from model (7)). If model (9) has feasible solution, $D M U_{l} \succ D M U_{k}$, otherwise no dominating relationship exists between them. 
Third, $r_{k}^{>}(u, w, v)=1+\left|R_{k}^{>}(u, w, v)\right|$ is thus obtained by adding the number of DMUs dominating $D M U_{k}$ and one.

Fourth, by running model (10), we could obtain the worst efficiency ranking of $D M U_{k}$.

\subsection{Ranking intervals of a DMU for both subsystems}

In this section, we discuss the ranking intervals of DMUs for two subsystems. we firstly give some definitions:

Definition 3. For every set of $\left(u_{r}, w_{d}, v_{i}\right), \quad r=1, \ldots s, d=1, \ldots D, i=1, \ldots, m$,

$$
\begin{aligned}
& R_{k}^{1>}(w, v)=\left\{l \in\{1, \ldots, n\} \mid E_{l}^{1}(w, v)>E_{k}^{1}(w, v)\right\} \\
& R_{k}^{2>}(u, w)=\left\{l \in\{1, \ldots, n\} \mid E_{l}^{2}(u, w)>E_{k}^{2}(u, w)\right\}
\end{aligned}
$$

$R_{k}^{1>}(w, v)$ contains the indexes of those other DMUs with larger efficiency scores for subsystem 1 than that of $D M U_{k}$ over feasible weights $(w, v) \cdot R_{k}^{2>}(u, w)$ contains the indexes of those other DMUs with larger efficiency scores for subsystem 2 than that of $D M U_{k}$ over feasible weights $(u, w)$. The corresponding efficiency rankings of $D M U_{k}$ for subsystem 1 and subsystem 2 are defined as $r_{k}^{1>}(w, v)=1+\left|R_{k}^{1>}(w, v)\right|$ and $r_{k}^{2>}(u, w)=1+\left|R_{k}^{2>}(u, w)\right|$, respectively

Definition 4. For every set of $\left(u_{r}, w_{d}, v_{i}\right), \quad r=1, \ldots s, d=1, \ldots D, i=1, \ldots, m$

$$
\begin{aligned}
& R_{k}^{1 \geq}(w, v)=\left\{l \in\{1, \ldots, n\} \backslash k \mid E_{l}^{1}(w, v) \geq E_{k}^{1}(w, v)\right\} \\
& R_{k}^{2 \geq}(u, w)=\left\{l \in\{1, \ldots, n\} \backslash k \mid E_{l}^{2}(u, w) \geq E_{k}^{2}(u, w)\right\}
\end{aligned}
$$

$R_{k}^{1 \geq}(w, v)$ contains the indexes of those other DMUs with no less efficiency scores for subsystem 1 than that of $D M U_{k}$ over feasible weights $(w, v)$. Similarly, $R_{k}^{2 \geq}(u, w)$ contains the indexes of those other DMUs with no less efficiency scores for subsystem 2 than that of $D M U_{k}$ over feasible weights $(u, w)$. The corresponding efficiency rankings of $D M U_{k}$ for subsystem 1 and subsystem 2 are defined as 
$r_{k}^{1 \geq}(w, v)=1+\left|R_{k}^{1 \geq}(w, v)\right|$ and $r_{k}^{2 \geq}(u, w)=1+\left|R_{k}^{2 \geq}(u, w)\right|$, respectively.

Proposition 3. The optimum of the following minimization problem is $r_{k}^{1 \mathrm{~min}}$, i.e. the best ranking of $D M U_{k}$ for subsystem 1 .

$$
\begin{array}{ll}
r_{k}^{1 \min }= & \min _{w, v} r_{k}^{1>}(w, v)= \\
& =\min _{w, v, p^{1}} 1+\sum_{l \neq k} p_{l}^{1} \\
\text { s.t. } & \sum_{d} w_{d} z_{d l} \leq \sum_{i} v_{i} x_{i l}+C p_{l}^{1} \quad l=1, \ldots, n,(l \neq k) \\
& \sum_{d} w_{d} z_{d k}=\sum_{i} v_{i} x_{i k}=1 \\
& p_{l}^{1} \in\{0,1\}, l \neq k \\
& w_{d}, v_{i}, d=1, \ldots, D ; i=1, \ldots, m
\end{array}
$$

In Model (11), the optimal value $\sum_{l \neq k} p_{l}^{1^{*}}$ in the objective function denotes the number of other DMUs with larger efficiency scores for subsystem 1 than that of $D M U_{k}$ over all feasible weights $(w, v)$. The constraints (11.1) and (11.2) assure that $D M U_{l}$ has larger efficiency score for subsystem 1 than that of $D M U_{k}$ over sets of all feasible weights $(w, v)$. The proof of this proposition is given in the Appendix.

Proposition 4. The optimum of the following maximization problem is $r_{k}^{1 \max }$, i.e. the worst ranking of $D M U_{k}$ for subsystem 1 .

$$
\begin{array}{ll}
r_{k}^{1 \max }=\max _{w, v} \quad r_{k}^{1 \geq}(w, v)= \\
& =\max _{w, v, p^{1}} 1+\sum_{l \neq k} p_{l}^{1} \\
\text { s.t. } & \sum_{i} v_{i} x_{i l} \leq \sum_{d} w_{d} z_{d l}+C\left(1-p_{l}^{1}\right) \quad l=1, \ldots, n,(l \neq k) \\
& \sum_{d} w_{d} z_{d k}=\sum_{i} v_{i} x_{i k}=1 \\
& p_{l}^{1} \in\{0,1\}, l \neq k \\
& w_{d}, v_{i}, d=1, \ldots, D ; i=1, \ldots, m
\end{array}
$$

The constraints (12.1) and (12.2) assure that $D M U_{l}$ has no less efficiency score for subsystem 1 than that of $D M U_{k}$. The optimal value $\sum_{l \neq k} p_{l}^{1^{*}}$ in the objective function denotes the number of DMUs with no less efficiency scores for subsystem 1 
than that of $D M U_{k}$. The proof of this proposition is given in the Appendix.

Similarly, according to the definition 3, the most favorable scenario of subsystem 2 for $D M U_{k}$ is that in which we have the maximum number of DMUs that perform worse in subsystem 2 or, equivalently, the minimum number of DMUs that perform better than $D M U_{k}$ in subsystem 2. The following definition establishes what we mean by the worst ranking $\left(r_{k}^{2 \min }\right)$ of a given $D M U_{k}$ in subsystem 2.

Proposition 5. The optimum of the following minimization problem is $r_{k}^{2 m i n}$, i.e. the best ranking of $D M U_{k}$ for subsystem 2 .

$$
\begin{array}{ll}
r_{k}^{2 \min } & =\min _{u, w, p^{2}} r_{k}^{2>}(u, w) \\
& =\min _{u, w, p^{2}} 1+\sum_{l \neq k} p_{l}^{2} \\
\text { s.t. } & \sum_{r} u_{r} y_{r l} \leq \sum_{d} w_{d} z_{d l}+C p_{l}^{2} \quad l=1, \ldots, n,(l \neq k) \\
& \sum_{r} u_{r} y_{r k}=\sum_{d} w_{d} z_{d k}=1 \\
& p_{l}^{2} \in\{0,1\}, l \neq k \\
& u_{r}, w_{d}, r=1, \ldots, s ; d=1, \ldots, D
\end{array}
$$

The optimal value $\sum_{l \neq k} p_{l}^{2^{*}}$ in the objective function denotes the number of other DMUs with larger efficiency scores for sub system 2 than that of $D M U_{k}$. The constraints (13.1) and (13.2) assure that $D M U_{l}$ has larger efficiency score for subsystem 2 than that of $D M U_{k}$. The proof is similar to Proposition 3.

According to the definition 4, the most unfavorable scenario of subsystem 2 for $D M U_{k}$ is that in which we have the minimum number of DMUs that perform worse or, equivalently, the maximum number of DMUs that perform no worse than $D M U_{k}$.

The following definition establishes what we mean by the worst ranking $\left(r_{k}^{\max }\right)$ of a given $D M U_{k}$ in subsystem 2 .

Proposition 6. The optimum of the following maximization problem is $r_{k}^{2 \max }$, i.e. the 
worst ranking of $D M U_{k}$ for subsystem 2 .

$$
\begin{array}{ll}
r_{k}^{2 \max }=\max _{u, w, p^{2}} r_{k}^{2 \geq}(u, w) \\
& =\max _{u, w, p^{2}} 1+\sum_{l \neq k} p_{l}^{2} \\
\text { s.t. } & \sum_{d} w_{d} z_{d l} \leq \sum_{r} u_{r} y_{r l}+C\left(1-p_{l}^{2}\right) \quad l=1, \ldots, n,(l \neq k) \\
& \sum_{r} u_{r} y_{r k}=\sum_{d} w_{d} z_{d k}=1 \\
& p_{l}^{2} \in\{0,1\}, l \neq k \\
& u_{r}, w_{d}, r=1, \ldots, s ; d=1, \ldots, D
\end{array}
$$

The constraint (14.1) assures that $D M U_{l}$ has no less efficiency score for subsystem 2 than that of $D M U_{k}$. The optimal value $\sum_{l \neq k} p_{l}^{2^{*}}$ in optimal objective function denotes the number of DMUs with no less efficiency scores for subsystem 2 than that of $D M U_{k}$. The proof is similar to Proposition 4.

The approach proposed above provides ranking intervals of $D M U_{k}$ for the overall system and both subsystems. On one hand, the ranking intervals provide information regarding the overall performance and the performance of two subsystems for different DMUs. For example, if $r_{k}^{1 \mathrm{~min}}<r_{l}^{1 \mathrm{~min}}$ and $r_{k}^{2 \mathrm{~min}}<r_{l}^{2 \mathrm{~min}}$, this means that $D M U_{k}$ may have better rankings for the overall system and both subsystems than that of $D M U_{l}$. On the other hand, it could be used to analyze the stability of the rankings for the overall system and both subsystems. For example, if $r_{k}^{\min }=r_{k}^{\max }$, then the ranking of $D M U_{k}$ is stable over all feasible weights and insensitive to the weights.

\section{Extension to multi-stage production process}

In this section, we extend the ranking interval model to multi-stage systems. In reality, many decision-making units (DMUs) have a multi-stage system structure (Kao 2014). For example, see Fig. 3 that shows a multi-stage process of $q$ sub-systems in series. The first sub-system uses inputs $\mathrm{X}_{\mathrm{i}}, \mathrm{i}=1, \ldots, \mathrm{m}$ supplied from outside to produce intermediate products $\mathrm{Z}_{\mathrm{d}}^{1}, \mathrm{~d} \in \mathrm{M}^{1}$ for sub-system 2 to use. In subsequent subsystems, every sub-system $\mathrm{p}$ consumes intermediate products $\mathrm{Z}_{\mathrm{d}}^{\mathrm{p}-1}, \mathrm{~d} \in \mathrm{M}^{\mathrm{p}-1}$, 
produced by its preceding process $\mathrm{p}-1$, to produce intermediate products $\mathrm{Z}_{\mathrm{d}}^{\mathrm{p}}, \mathrm{d} \in \mathrm{M}^{\mathrm{p}}$ for the succeeding process $\mathrm{p}+1$ to use. For the last sub-system qq, it consumes intermediate products $\mathrm{Z}_{\mathrm{d}}^{\mathrm{q}-1}, \mathrm{~d} \in \mathrm{M}^{\mathrm{q}-1}$ to produce final outputs $\mathrm{Y}_{\mathrm{r}}, \mathrm{r}=1, \ldots, \mathrm{s}$.

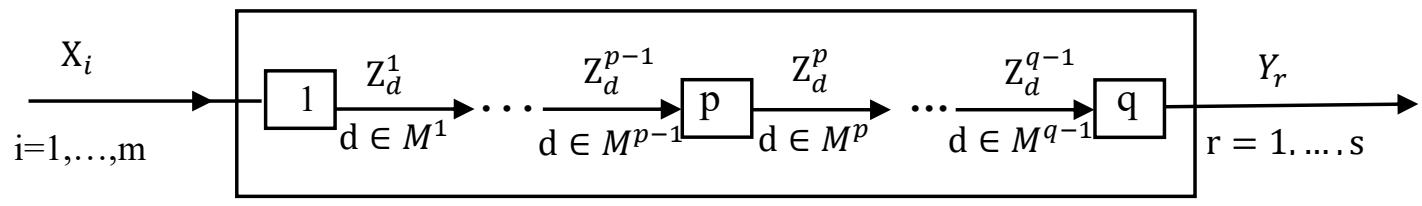

Fig. 3 Multi-stage production process

According to Kao (2014), the efficiency for the overall system and sub-systems are defined as follows:

$$
\begin{aligned}
E_{k} & =\frac{\sum_{r=1}^{s} u_{r} Y_{r k}}{\sum_{i=1}^{m} v_{i} X_{i k}} \\
\mathrm{E}_{\mathrm{k}}^{1} & =\frac{\sum_{\mathrm{d} \in \mathrm{M}^{1} \mathrm{w}_{\mathrm{d}} \mathrm{z}_{\mathrm{dk}}^{1}}}{\sum_{\mathrm{i}=1}^{\mathrm{m}} \mathrm{v}_{\mathrm{i}} \mathrm{X}_{\mathrm{ik}}} \\
\mathrm{E}_{\mathrm{k}}^{\mathrm{p}} & =\frac{\sum_{\mathrm{d} \in \mathrm{M}} \mathrm{p} \mathrm{w}_{\mathrm{d}} \mathrm{z}_{\mathrm{dk}}^{\mathrm{p}}}{\sum_{\mathrm{d} \in \mathrm{M}} \mathrm{p}-1 \mathrm{w}_{\mathrm{d}} \mathrm{z}_{\mathrm{dk}}^{\mathrm{p}-1}}, \mathrm{p}=2, \ldots, \mathrm{q}-1 \\
\mathrm{E}_{k}^{\mathrm{q}} & =\frac{\sum_{r=1}^{s} u_{r} Y_{r k}}{\sum_{\mathrm{d} \in \mathrm{M}} \mathrm{q}-1 \mathrm{w}_{\mathrm{d}} \mathrm{z}_{\mathrm{dk}}^{\mathrm{q}-1}}
\end{aligned}
$$

where $u_{r}(r=1, \ldots, s), w_{d}(d \in M)$ and $v_{i}(i=1, \ldots, m)$ are the output weights, intermediate weights and input weights, respectively. The system efficiency for the overall system with a multiple-stage structure is the product of the efficiencies for all sub-systems' efficiencies.

Similar to section 2, to obtain the ranking intervals for DMUs with a multi-stage production system, we firstly need to give some definitions.

Definition 6. For every set of $\left(u_{r}, w_{d}, v_{i}\right), r=1, \ldots, s ; d \in M ; i=1, \ldots, m$, $R_{k}^{>}(u, w, v)=\left\{\begin{array}{l|l}l \epsilon\{1, \ldots, n\} & \begin{array}{c}E_{l}^{p}(u, w, v)>E_{k}^{p}(u, w, v), p=1, \ldots, q \text { or } \\ E_{l}^{t}(u, w, v)>E_{k}^{t}(u, w, v), t \epsilon\{1, \ldots, q\} \text { and } \\ E_{l}^{t 1}(u, w, v) \geq E_{k}^{t 1}(u, w, v) \\ , t 1 \neq t \text { and } t 1 \epsilon\{1, \ldots, q\}\end{array}\end{array}\right\}$

$R_{k}^{>}(u, w, v)$ contains the indexes of those other DMUs with larger efficiency scores for all subsystems, or with larger efficiency scores for one sub-system and no less efficiency scores for other sub systems than that of $D M U_{k}$. Similar to the 
Definition 1 for the ranking intervals of two-stage process, $R_{k}^{>}(u, w, v)$ contains the indexes of $D M U_{1}(l=1, \ldots, n, l \neq k)$ that dominate $D M U_{k}$.

Definition 7. For every set of $\left(u_{r}, w_{d}, v_{i}\right), \quad r=1, \ldots s, d=1, \ldots D, i=1, \ldots, m$,

$$
R_{k}^{\geq}(u, w, v)=\left\{l \in\{1, \ldots, n\} \backslash k \mid E_{l}^{p}(u, w, v) \geq E_{k}^{p}(u, w, v), p=1, \ldots, q\right\}
$$

$R_{k}^{\geq}(u, w, v)$ contains the indexes of those other DMUs with no less efficiency scores for all subsystems than that of $D M U_{k}$. The corresponding efficiency rankings for $D M U_{k}$ are defined as $r_{k}^{>}(u, w, v)=1+\left|R_{k}^{>}(u, w, v)\right|$ and $r_{k}^{\geq}(u, w, v)=1+\left|R_{k}^{\geq}(u, w, v)\right|$, respectively. We can see the following proposition as an extension of Proposition 1 to multistage systems:

Proposition 7. The optimum of the minimization problem

$$
\begin{aligned}
& \operatorname{Min}_{u, w, v, t^{1}, \ldots, t} \sum_{p=1}^{q} t_{l}^{p} \\
& \text { s.t. } \sum_{r=1}^{s} u_{r} Y_{r l} \leq \sum_{\mathrm{d} \in \mathrm{M}^{\mathrm{q}-1}} \mathrm{w}_{\mathrm{d}} \mathrm{Z}_{\mathrm{dl}}^{\mathrm{q}-1}+C t_{l}^{q}, l=1, \ldots, n(l \neq k)(19.1) \\
& \sum_{\mathrm{d} \in \mathrm{M}^{\mathrm{p}}} \mathrm{w}_{\mathrm{d}} \mathrm{z}_{\mathrm{dl}}^{\mathrm{p}} \leq \sum_{\mathrm{d} \in \mathrm{M}^{\mathrm{p}-1}} \mathrm{w}_{\mathrm{d}} \mathrm{z}_{\mathrm{dl}}^{\mathrm{p}-1}+C t_{l}^{p}, p=2, \ldots, q-1, l=1, \ldots, n(l \neq k) \\
& \sum_{\mathrm{d} \in \mathrm{M}^{1}} \mathrm{w}_{\mathrm{d}} \mathrm{Z}_{\mathrm{dl}}^{1} \leq \sum_{\mathrm{i}=1}^{\mathrm{m}} \mathrm{v}_{\mathrm{i}} \mathrm{X}_{\mathrm{il}}+C t_{l}^{1}, l=1, \ldots, n(l \neq k) \\
& \sum_{r=1}^{s} u_{r k}=\sum_{d \in M^{q-1}} w_{d} Z_{d k}^{q-1}=1
\end{aligned}
$$

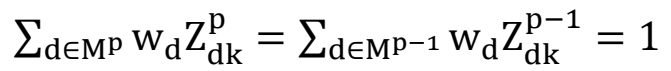

$$
\begin{aligned}
& \sum_{\mathrm{d} \in \mathrm{M}^{1}} \mathrm{~W}_{\mathrm{d}} \mathrm{Z}_{\mathrm{dk}}^{1}=\sum_{i=1}^{m} v_{i} X_{i k}=1 \\
& t_{l}^{1}, t_{l}^{2}, \ldots, t_{l}^{q} \in\{0,1\}, l \neq k \\
& u_{r}, w_{d}, v_{i} \geq 0, \forall r, d, i
\end{aligned}
$$

is to identify the DMUs with strictly larger efficiency scores for all subsystems than that of $D M U_{k}$. The constraints (19.1) - (19.6) assure that $D M U_{l}$ has larger efficiency scores for all subsystems than that of $D M U_{k}$. If $E_{l}^{p}(u, w, v)>E_{k}^{p}(u, w, v), p=$ $1, \ldots, q$, then the optimal value in the objective function is $q$, i.e., $\sum_{p=1}^{q} t_{l}^{p *}=q$ $\sum_{p=1}^{q} t_{l}^{p *}=q$. If $D M U_{l}$ has larger efficiency scores for some sub-systems while it 
has no less efficiency scores for other sub-systems than that of $D M U_{k}$, then $1 \leq$ $\sum_{p=1}^{q} t_{l}^{p *} \leq q-1$ holds; if $D M U_{l}$ has no larger efficiency scores for all sub-systems than that of $D M U_{k}$, i.e., $E_{l}^{p}(u, w, v) \leq E_{k}^{p}(u, w, v), p=1, \ldots, q$, then $\sum_{p=1}^{q} t_{l}^{p *}=0$ holds. Thus, the model has a feasible solution.

Therefore, the optimal value in the objective function may be clarified into three categories: $0,[1, \mathrm{q}-1]$ or q. (1) If $\sum_{p=1}^{q} t_{l}^{p *}=q$ holds in model (19), then $D M U_{l}$ dominates $D M U_{k}$.(2) If $\sum_{p=1}^{q} t_{l}^{p *}=0$ holds, then $D M U_{l}$ does not dominate $D M U_{k}$ as $D M U_{l} \prec D M U_{k}$ or $D M U_{l} \approx D M U_{k}$. (3) If $1 \leq \sum_{p=1}^{q} t_{l}^{p *} \leq q-1$ holds, $D M U_{l}$ dominates $D M U_{k}\left(D M U_{l} \succ D M U_{k}\right)$ or no dominating relationship may exist between $D M U_{l}$ and $D M U_{k}$. Therefore, by applying model (19), we could identify the DMUs dominating $D M U_{k}$ if $\sum_{p=1}^{q} t_{l}^{p *}=q$ holds. However, we could not identify whether $D M U_{l}$ dominates $D M U_{k}$ or no dominating relationship between them if $1 \leq \sum_{p=1}^{q} t_{l}^{p *} \leq q-1$ holds. To further identify whether $D M U_{l}$ dominates $D M U_{k}$ if $1 \leq \sum_{p=1}^{q} t_{l}^{p *} \leq q-1$ holds, similar models like model (8) and model (9) could be proposed.

For example, if $\sum_{p=1}^{q} t_{l}^{p *}=1$ holds and $E_{l}^{1}>E_{k}^{1}$ and $E_{l}^{p}(u, w, v) \leq$ $E_{k}^{p}(u, w, v), p=2, \ldots, q$ (From model (19)), then the following model could further identify whether $D M U_{l}$ satisties $E_{l}^{1}>E_{k}^{1}$ and $E_{l}^{p}(u, w, v)=E_{k}^{p}(u, w, v), p=$ $2, \ldots, q$

$$
\begin{aligned}
& \operatorname{Min}_{u, w, v} C \\
& \text { s.t. } \sum_{r=1}^{s} u_{r} Y_{r l} \geq \sum_{\mathrm{d} \in \mathrm{M}^{\mathrm{q}-1}} \mathrm{w}_{\mathrm{d}} \mathrm{Z}_{\mathrm{dl}}^{\mathrm{q}-1}, l=1, \ldots, n(l \neq k)(20.1) \\
& \sum_{\mathrm{d} \in \mathrm{M}^{\mathrm{p}}} \mathrm{w}_{\mathrm{d}} \mathrm{z}_{\mathrm{dl}}^{\mathrm{p}} \geq \sum_{\mathrm{d} \in \mathrm{M}^{\mathrm{p}-1}} \mathrm{w}_{\mathrm{d}} \mathrm{z}_{\mathrm{dl}}^{\mathrm{p}-1}, p=2, \ldots, q-1, l=1, \ldots, n(l \neq k) \\
& \sum_{\mathrm{d} \in \mathrm{M}^{1}} \mathrm{w}_{\mathrm{d}} \mathrm{Z}_{\mathrm{dl}}^{1}>\sum_{\mathrm{i}=1}^{\mathrm{m}} \mathrm{v}_{\mathrm{i}} \mathrm{X}_{\mathrm{il}}, l=1, \ldots, n(l \neq k) \\
& \sum_{r=1}^{s} u_{r k}=\sum_{d \in M^{q-1}} w_{d} Z_{d k}^{q-1}=1
\end{aligned}
$$

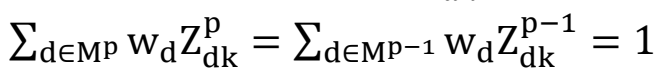




$$
\begin{gathered}
\sum_{\mathrm{d} \in \mathrm{M}^{1}} \mathrm{w}_{\mathrm{d}} \mathrm{Z}_{\mathrm{dk}}^{1}=\sum_{i=1}^{m} v_{i} X_{i k}=1(20.6) \\
u_{r}, w_{d}, v_{i} \geq 0, \forall r, d, i
\end{gathered}
$$

The objective value of $C$ is constant. $D M U_{k}$ is the evaluated DMU. The constraints (20.1) - (20.6) assure that $D M U_{l}$ has larger efficiency score for subsystem 1 and no less efficiency score for other subsystems than that of $D M U_{k}$. If model (20) has a feasible solution, then $E_{l}^{1}>E_{k}^{1}$ and $E_{l}^{p}(u, w, v)=E_{k}^{p}(u, w, v), p=2, \ldots, q$. In such case, $D M U_{l}$ dominates $D M U_{k}$. If model (20) has no feasible solution, then $E_{l}^{1}>E_{k}^{1}$ and $E_{l}^{p}(u, w, v)<E_{k}^{p}(u, w, v), p=2, \ldots, q$. In such case, there exists no dominating relation between $D M U_{l}$ and $D M U_{k}$. Thus, this model could identify the DMUs dominating $D M U_{k}$. Similar models could be developed to identify whether $D M U_{l}$ dominates $D M U_{k}$ when the optimal value of model (19) is between 1 and $\mathrm{q}-1$, i.e., $\quad 1 \leq \sum_{p=1}^{q} t_{l}^{p *} \leq q-1$.

The most unfavorable scenario for $D M U_{k}$ is that in which we have the minimum number of DMUs whose all subsystems perform worse or, equivalently, the maximum number of DMUs whose all subsystems perform no worse than $D M U_{k}$ by definition 7. The following proposition establishes what we mean by the worst ranking of a given $D M U_{k}$.

Proposition 8. In the following model, the optimum of the maximization problem, i.e. $r_{k}^{\max }$, is the worst efficiency ranking of $D M U_{k}$ considering each DMU has a multistage production system.

$$
\max _{u, w, v} r_{k}^{\geq}(u, w, v)=\max _{u, w, v, t^{1}, \ldots, t^{q}} 1+\sum_{l \neq k}^{q} \prod_{p=1}^{q} t_{l}^{p}
$$

s.t. $\sum_{\mathrm{d} \in \mathrm{M}^{\mathrm{q}-1}} \mathrm{w}_{\mathrm{d}} \mathrm{Z}_{\mathrm{dl}}^{\mathrm{q}-1} \leq \sum_{r=1}^{s} u_{r} Y_{r l}+C\left(1-t_{l}^{q}\right), l=1, \ldots, n(l \neq k)(21.1)$

$\sum_{\mathrm{d} \in \mathrm{M}^{\mathrm{p}-1}} \mathrm{w}_{\mathrm{d}} \mathrm{Z}_{\mathrm{dl}}^{\mathrm{p}-1} \leq \sum_{\mathrm{d} \in \mathrm{M}^{\mathrm{p}}} \mathrm{w}_{\mathrm{d}} \mathrm{Z}_{\mathrm{dl}}^{\mathrm{p}}+C\left(1-t_{l}^{p}\right), p=2, \ldots, q-1, l=1, \ldots, n(l \neq k)$ 


$$
\begin{aligned}
& \sum_{\mathrm{i}=1}^{\mathrm{m}} \mathrm{v}_{\mathrm{i}} \mathrm{X}_{\mathrm{il}} \leq \sum_{\mathrm{d} \in \mathrm{M}^{1}} \mathrm{w}_{\mathrm{d}} \mathrm{Z}_{\mathrm{dl}}^{1}+C\left(1-t_{l}^{1}\right), l=1, \ldots, n(l \neq k) \\
& \sum_{r=1}^{s} u_{r k}=\sum_{d \in M^{q-1}} w_{d} Z_{d k}^{q-1}=1 \\
& \sum_{\mathrm{d} \in \mathrm{M}^{\mathrm{p}}} \mathrm{w}_{\mathrm{d}} \mathrm{z}_{\mathrm{dk}}^{\mathrm{p}}=\sum_{\mathrm{d} \in \mathrm{M}^{\mathrm{p}-1} \mathrm{w}_{\mathrm{d}} \mathrm{Z}_{\mathrm{dk}}^{\mathrm{p}-1}=1}^{m} v_{\mathrm{d} \in \mathrm{M}^{1}} \mathrm{w}_{\mathrm{d}} \mathrm{z}_{\mathrm{dk}}^{1}=\sum_{i=1}^{m} v_{i} X_{i k}=1(21.6) \\
& t_{l}^{1}, t_{l}^{2}, \ldots, t_{l}^{q} \in\{0,1\}, l \neq k \\
& u_{r}, w_{d}, v_{i} \geq 0, \forall r, d, i
\end{aligned}
$$

The constraints (21.1)- (21.6) assure that $D M U_{l}$ has no less efficiency scores for all subsystems than that of $D M U_{k}$. The optimal value $\sum_{l \neq k} \prod_{p=1}^{q} t_{l}^{p}$ in the objective function denotes the number of DMUs with no less efficiency scores for both subsystems than that of $D M U_{k}$. When the efficiency scores of $D M U_{l}$ for all subsystems are no less than that of $D M U_{k}, \prod_{p=1}^{q} t_{l}^{p *}$ is one, otherwise, $\prod_{p=1}^{q} t_{l}^{p *}$ is zero. The model is a mixed-integer program and non-linear. We solve it by using YALMIP toolbox in MATLAB.

\section{Empirical illustration}

To illustrate the proposed approach of ranking intervals for two-stage production systems, we use the following two examples.

\subsection{An illustration example}

For illustration purpose, we use a numerical example with two inputs, one intermediate and two outputs as used in Kao and Hwang (2010) and reproduced in Table 1.

Table 1: Numerical data set (Example 1)

\begin{tabular}{cccccc}
\hline DMU & $\mathrm{x} 1$ & $\mathrm{x} 2$ & $\mathrm{z}$ & $\mathrm{y} 1$ & $\mathrm{Y} 2$ \\
\hline P1 & 1 & 2 & 1.6 & 2 & 3 \\
P2 & 2 & 1 & 1 & 3 & 3 \\
P3 & 4 & 5 & 0.67 & 2 & 4 \\
P4 & 5 & 5 & 0.6 & 1 & 2 \\
\hline
\end{tabular}

Table 2 reports the results of ranking intervals based on the proposed approach and compare it with Salo and Punkka (2011)'s approach. The ranking intervals considering 
the two-stage production system (based on our approach) are shown from Column 2 to 7 while the ranking intervals treating the production as a "Black Box" (based on Salo and Punkka (2011)'s approach) are shown in Column 8 and 9.

Table 2: Ranking intervals (Example 1)

\begin{tabular}{ccccccccc}
\hline & \multicolumn{9}{c}{ Our approach } & \multicolumn{3}{c}{$\begin{array}{c}\text { Salo \& Punkka } \\
\text { DMU }\end{array}$} & \multicolumn{9}{c}{ (2011)'s approach } \\
\cline { 2 - 10 } & $r_{k}^{\min }$ & $r_{k}^{\max }$ & $r_{k}^{1 \min }$ & $r_{k}^{1 \max }$ & $r_{k}^{2 \min }$ & $r_{k}^{2 \max }$ & $r_{k}^{\min }$ & $r_{k}^{\max }$ \\
\hline P1 & 2 & 2 & 1 & 2 & 3 & 4 & 1 & 2 \\
P2 & 1 & 1 & 1 & 2 & 3 & 4 & 1 & 3 \\
P3 & 2 & 2 & 3 & 3 & 1 & 2 & 2 & 4 \\
P4 & 3 & 3 & 4 & 4 & 1 & 2 & 3 & 4 \\
\hline
\end{tabular}

This example is the situation that the ranking intervals based on our approach may be narrower than that of Salo and Punkka (2011)'s approach. Thus, the rankings may be not very sensitive to the weights based on our approach. As shown in Column 2 and 3 in Table 2, it could be found that DMU (P1) and DMU (P3) always ranks 2 regardless what weights are chosen. DMU (P2) always ranks 1 and DMU (P4) always ranks 3 regardless of what weights are chosen. Therefore, we can conclude that the rankings of these DMUs do not depend on the choice of DEA weights that the DMUs make. Hence, there is no difference between the best ranking and the worst ranking. However, the ranking intervals based on Salo and Punkka (2011)'s approach are wider. For example, DMU (P1) has the best ranking of 1 and the worst ranking of 2 if we do not consider the two-stage structure.

This example also shows that the best rankings based on our approach are no less than that of Salo and Punkka (2011)'s approach.Similarly, the worst rankings based on our approach are no larger than that of Salo and Punkka (2011)'s approach. For example, regardless what weights are chosen, DMU (P2) always ranks 1 based on our approach. However, the best and worst rankings based on Salo and Punkka (2011)'s approach are 1 and 3, respectively. It is similar to other three DMUs. This may be due to the fact that 
if a DMU dominates the other DMU, then it has larger efficiency scores for both subsystems, or larger efficiency score for one subsystem and no less efficiency score for the other subsystem (see Definition 1).

The ranking intervals also provide information to rank the DMUs in the overall system and both two subsystems. If we consider the two-stage production system, DMU (P2) always ranks 1 in the overall system regardless what weights are chosen. So DMU (P2) has the best performance in the overall system. But if we do not consider the twostage system structure, DMU (P1) and DMU (P2) may have good performance as they may rank 1 . The result is different from our approach because they do not consider the two-stage production structure. We believe that our approach produces a more realistic results since it considers the internal structure of DMUs and could be applied to real two-stage production processes. In subsystem 1, DMU (P1) and DMU (P2) have the best ranking of 1, accordingly, they are the best DMUs in subsystem 1. Similarly, in the subsystem 2, DMU (P3) and DMU (P4) are the best DMUs as their best ranking could attain 1.

\subsection{An application of non-life insurance companies}

In this section, we take the data set of 24 non-life insurance companies from (Kao \& Hwang, 2008). These non-life insurance companies' whole production system has a typical two-stage structure. The production system is divided into two subsystems: premium acquisition and profit generation. These companies are evaluated by using two inputs, two intermediates, and two outputs.

Table 3: Ranking intervals for each non-life insurance company (Example 2)

\begin{tabular}{cccccc} 
& NO. & \multicolumn{2}{c}{ Our approach } & \multicolumn{2}{c}{ Salo \& Punkka (2011)'s } \\
DMU & & $r_{k}^{\min }$ & $r_{k}^{\max }$ & $r_{k}^{\min }$ & $r_{k}^{\max }$ \\
\cline { 3 - 6 } & & 6 & 12 & 2 & 17 \\
\hline Taiwan Fire & 1 & 11 & 19 & 1 & 20 \\
Chung Kuo & 2 & 20 & 23 & 2 & 23 \\
Tai Ping & 3 & 19 & 21 & 8 & 24 \\
China Mariners & 4 & 3 & 15 & 1 & 13 \\
Fubon & 5 & 9 & 16 & 6 & 23 \\
Zurich & 6 & &
\end{tabular}




\begin{tabular}{cccccc}
\hline Taian & 7 & 5 & 11 & 9 & 19 \\
Ming Tai & 8 & 4 & 10 & 8 & 20 \\
Central & 9 & 5 & 12 & 15 & 22 \\
The First & 10 & 8 & 13 & 5 & 19 \\
Kuo Hua & 11 & 7 & 13 & 9 & 24 \\
Union & 12 & 7 & 17 & 1 & 21 \\
Shingkong & 13 & 3 & 8 & 6 & 23 \\
South China & 14 & 8 & 15 & 10 & 20 \\
Cathay Century & 15 & 2 & 7 & 1 & 13 \\
Allianz President & 16 & 11 & 17 & 9 & 20 \\
Newa & 17 & 2 & 4 & 3 & 14 \\
AIU & 18 & 10 & 14 & 12 & 21 \\
North America & 19 & 1 & 3 & 2 & 21 \\
Federal & 20 & 2 & 2 & 2 & 16 \\
Royal \& Sun & 21 & & & 13 & 23 \\
Alliance & & 7 & 7 & 1 & 12 \\
Asia & 22 & 1 & 21 & 6 & 24 \\
AXA & 23 & 12 & 6 & 14 & 24 \\
\hline Mitsui Sumitomo & 24 & 6 & &
\end{tabular}

Table 3 reports the ranking intervals of each DMU based on our approach and Salo \& Punkka (2011)'s approach. Column 5 and column 6 in Table 3 report the DMUs' ranking intervals treating the production system as a "Black Box", which are represented graphically red in Fig.4. Column 3 and column 4 in Table 3 report the DMUs' ranking intervals when considering the inner production structure, which are represented graphically blue in Fig.4. 


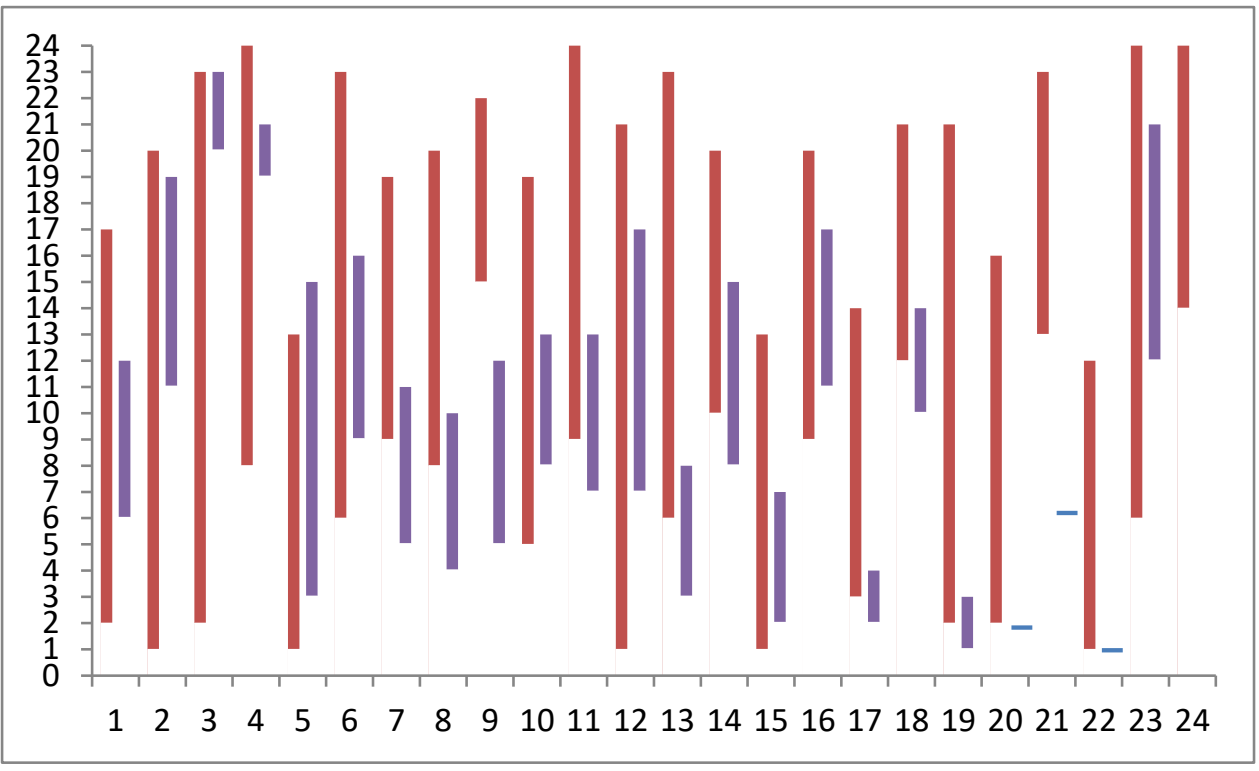

Fig. 4 Ranking intervals for each non-life insurance company considering and without considering the two-stage production system

From Fig.4, we can find that if we do not consider the two-stage production structure, the best DMUs are Chung Kuo (DMU 2), Fubon (DMU 5), Union (DMU 12), Cathay Century (DMU 15), and Asia (DMU 22) as they have the best ranking of 1 . Though these DMUs may rank 1, they have wide ranking ranges. Among these five DMUs, Asia (DMU 22) has the best performance as it has the narrowest ranking intervals as well as the best ranking of 1 . The worst DMUs are China Mariners (DMU 4), Kuo Hua (DMU 11), AXA (DMU 23) and Mitsui Sumitomo (DMU 24) as their worst rankings are 24. Among these four DMUs, the best ranking of AXA (DMU 23) is 14, which is larger than that of other three DMUs. Thus, AXA (DMU 23) is the worst DMU.

The best performers (or worst performers) based on our approach may not be the same as that of Salo and Punkka (2011)'s approach. The blue bar char in Fig.4 reports the DMUs' ranking intervals when the two-stage structure is considered. Fig.4 shows that only North America (DMU 19) and Asia (DMU 22) are ranked 1 as they all have the best ranking of 1. But Asia (DMU 22) has the narrowest ranking interval as it ranks 1 regardless of what weights are chosen, so it is the best performer. Tai Ping (DMU 3) is the worst DMU as it has the worst ranking of 23. Besides, we can compare some 
DMUs over sets of all feasible weights. For example, Tai Ping (DMU 3) has a best ranking of 20 and a worst ranking of 23, while Ming Tai (DMU 8) has a best ranking of 4 and a worst ranking of 10 . That is, $r_{8}^{\min }<r_{3}^{\min }$ and $r_{8}^{\max }<r_{3}^{\min }<r_{3}^{\max }$. Hence, Ming Tai (DMU 8) always performs better than Tai Ping (DMU 3) regardless of the choice of the weights.

When the two-stage structure is considered, the ranking intervals may be narrower based on our approach than that of Salo and Punkka (2011)'s approach. For example, as shown in Column 3 and 4 in Table 3, it could be found that Allianz President (DMU 16) has the best ranking of 2 and the worst ranking of 20 . However, as shown in Column 5 and 6 in Table 3, it has the best ranking of 10 and the worst ranking of 22 over all feasible weights. Thus, the ranking intervals based on Salo and Punkka (2011)'s approach are wider.

Table 4: Ranking intervals for two subsystems

\begin{tabular}{|c|c|c|c|c|c|}
\hline \multirow{2}{*}{ DMU } & \multirow{2}{*}{ NO. } & \multicolumn{2}{|c|}{$\begin{array}{l}\text { Ranking intervals for } \\
\text { subsystem } 1\end{array}$} & \multicolumn{2}{|c|}{$\begin{array}{l}\text { Ranking intervals for } \\
\text { subsystem } 2\end{array}$} \\
\hline & & $r_{k}^{1 \min }$ & $r_{k}^{1 \max }$ & $r_{k}^{2 \min }$ & $r_{k}^{2 \max }$ \\
\hline Taiwan Fire & 1 & 1 & 12 & 4 & 19 \\
\hline Chung Kuo & 2 & 1 & 18 & 5 & 21 \\
\hline Tai Ping & 3 & 9 & 22 & 1 & 23 \\
\hline China Mariners & 4 & 7 & 24 & 9 & 23 \\
\hline Fubon & 5 & 3 & 23 & 1 & 15 \\
\hline Zurich & 6 & 2 & 23 & 12 & 20 \\
\hline Taian & 7 & 7 & 23 & 5 & 19 \\
\hline Ming Tai & 8 & 8 & 23 & 5 & 21 \\
\hline Central & 9 & 1 & 19 & 12 & 24 \\
\hline The First & 10 & 4 & 24 & 3 & 14 \\
\hline Kuo Hua & 11 & 7 & 24 & 11 & 24 \\
\hline Union & 12 & 1 & 23 & 3 & 17 \\
\hline Shingkong & 13 & 5 & 21 & 2 & 23 \\
\hline South China & 14 & 9 & 23 & 6 & 18 \\
\hline Cathay Century & 15 & 1 & 19 & 2 & 13 \\
\hline Allianz President & 16 & 2 & 20 & 10 & 22 \\
\hline Newa & 17 & 9 & 24 & 1 & 12 \\
\hline AIU & 18 & 3 & 21 & 12 & 23 \\
\hline North America & 19 & 1 & 20 & 3 & 23 \\
\hline
\end{tabular}




\begin{tabular}{clllll} 
Federal & 20 & 2 & 24 & 2 & 19 \\
Royal \& Sun Alliance & 21 & 7 & 24 & 8 & 22 \\
Asia & 22 & 5 & 24 & 1 & 17 \\
AXA & 23 & 3 & 16 & 6 & 24 \\
Mitsui Sumitomo & 24 & 1 & 24 & 6 & 24 \\
\hline
\end{tabular}

As for two subsystems, Table 4 shows the subsystem's ranking intervals of each insurance company. The third and fourth columns in Table 4 are the best and worst rankings for subsystem 1. The fifth and sixth columns in Table 4 are the best and worst rankings for subsystem 2. It shows that Taiwan Fire (DMU 1), Chung Kuo (DMU 2), Central (DMU 9), Union (DMU 12), Cathay Century (DMU 15), North America (DMU 19) and Mitsui Sumitomo (DMU 24) are the best performers in subsystem 1. As Taiwan Fire (DMU 1) has the narrowest ranking interval, it is the best DMU for subsystem 1. It can be seen that Taiwan Fire (DMU 1) has the best ranking of 4 and the worst ranking of 19 in subsystems 2 , so it does not perform very well in subsystem 2 . Therefore, the reason that Taiwan Fire (DMU 1)'s overall ranking is good is due to its well performance in subsystem 1. As for the subsystem 2, Tai Ping (DMU 3), Fubon (DMU 5), Newa (DMU 17), Asia (DMU 22) are the best DMUs as their best rankings are all 1. Central (DMU 9), Kuo Hua (DMU 11), AXA (DMU 23) and Mitsui Sumitomo (DMU 24) are the worst DMUs as their worst rankings can attain 24.

\subsection{An application to Chinese commercial banks}

In the DEA literature for bank and bank branch evaluation, about the input and output selections for bank efficiency measures, there are three common approaches, production approach, profitability approach and intermediation approach (Paradi et al. 2011). The production approach is used to investigate the ability of a branch/bank for using inputs - capital and labor to produce outputs - transaction services (e.g. Berger and Humphrey (1997); Fukuyama and Weber (2009)).The intermediation approach evaluates the operation of a branch as an entity using monetary assets as inputs to make loans and investments as outputs (e.g. Maudos et al. 2002; Chen et al. 2005). The profitability approach measures a branch's profitability based on expenses as inputs and 
revenues as outputs (Drake et al. (2006); Pasiouras, 2008a). Wang et al. 2014 considers the internal structure of a bank, and divides the producing process into two sub-systems, where deposits are taken as intermediate measure. In the first stage, fixed assets and labor are used to produce deposits; in the second stage, deposits are used to produce interest income and non-interest income. According to the available data and the characters of Chinese commercial banks, in this paper, the production approach is selected to choose the indexes.

In the first stage, deposit-producing stage, fixed assets and employee expenses are used to produce deposits. Fixed assets refer to the asset value of physical capital, and employee expenses refers to the payment to full-time employees hired. In the second stage, profit-earning stage, the deposits are used to produce interest income and nonincome interest. Deposits includes current deposits and time deposits, interest incomes refer to incomes that are primarily derived from loans and non-interest incomes include fees, commissions, investment and other business income. Our study considers 16 stock-listed commercial banks in China. They could be divided into three kinds, that is, State-owned commercial banks (SOB), National joint-stock commercial banks (NJB) and city commercial banks (CB). Among 16 main commercial banks, China Construction Bank (CCB), Industrial and Commercial Bank of China (ICBC), Bank of China (BOC), Agriculture Bank of China (ABC) and Bank of Communications (BOCOM) belong to State-owned commercial Banks; China Merchants Bank (CMB), China CITIC Bank (CNCB), China Minsheng Bank (CMBC), Industrial Bank (IB), Shanghai Pudong Development Bank (SPDB), China Everbright Bank (CEB), Hua Xia Bank (HXB), and Ping An Bank (PAB) belong to National joint-venture commercial banks (NJB) contain; Beijing Bank (BJB), Nanjing Bank (NJB) and Ningbo Bank (NBB) belong to City commercial banks. The data of Chinese commercial banks are derived from Bank-scope resource package produced by Bureau Van Dijk (BVD), Yearly Statistics Book of China's Finance and the annual reports of the banks. The descriptive statistics of the inputs, intermediate measures, and outputs of these banks are presented in Table 5. 
Table 5: Descriptive statistics of the inputs, intermediate measures, and outputs of Chinese

commercial banks

\begin{tabular}{ccccc}
\hline Variables & Mean & Standard deviation & Maximum & Minimum \\
\hline Fixed Assets & 677.05 & 803.02 & 2436.19 & 54.19 \\
Labors & 139.6 & 127.28 & 399.02 & 18.63 \\
Deposits & 56177.15 & 59528.32 & 178253.02 & 5114.05 \\
Interest Income & 695.06 & 655.45 & 2040.45 & 53.91 \\
non-interest income & 2875.72 & 2469.74 & 7914.80 & 337.54 \\
\hline
\end{tabular}

Table 6 reports the results of ranking intervals based on the proposed approach. The ranking intervals for the overall system are shown in Column 3 and 4. And, the ranking intervals for two sub-systems are shown in Column 5 to 8 .

The best performers (or worst performers) based on our approach could be identified. From Table 6, it could be seen that CMBC (DMU 4) and CMB (DMU 5) may be best DMUs as they both have the best ranking of 1. But CMBC (DMU 4) has the narrowest ranking interval as it ranks 1 regardless of what weights are chosen, so it is the best performer. $\mathrm{ABC}$ (DMU 7) is the worst DMU as it has the worst ranking of 16. Besides, we can compare some DMUs over sets of all feasible weights. For example, CCB (DMU 11) has a best ranking of 7 and a worst ranking of 12, while CMB (DMU 5) has a best ranking of 1 and a worst ranking of 6 . That is, $r_{5}^{\min }<r_{11}^{\min }$ and $r_{5}^{\max }<$ $r_{11}^{\min }<r_{11}^{\max }$. Hence, CMB (DMU 5) always performs better than CCB (DMU 11) regardless of the choice of the weights.

The ranking intervals also provide information to rank the DMUs in the overall system and both two subsystems. CMBC (DMU 4) and CMB (DMU 5) may rank 1 in the overall system, so they have the best performance in the overall system. In subsystem 1, PAB (DMU 1), BOCOM (DMU 8), ICBC (DMU 9), CNCB (DMU 13) and BJB (DMU 14) have the best ranking of 1, accordingly, they are the best DMUs in subsystem 1. Similarly, in the subsystem 2, CMBC (DMU 4) and CMB (DMU 5) are the best DMUs as their best ranking could attain 1. CMBC (DMU 4) may be the best 
DMU in the overall system as its good performance in subsystem 2 (it has best ranking of 1 and worst ranking of 8 in subsystem 2)

Table 6: Ranking intervals for Chinese commercial banks based on our approach

\begin{tabular}{cccccccc}
\hline & & \multicolumn{2}{c}{$\begin{array}{c}\text { Ranking intervals for } \\
\text { overall system }\end{array}$} & \multicolumn{2}{c}{$\begin{array}{c}\text { Ranking intervals for } \\
\text { subsystem } 1\end{array}$} & \multicolumn{2}{c}{$\begin{array}{c}\text { Ranking intervals for } \\
\text { subsystem } 2\end{array}$} \\
\cline { 3 - 8 } & & $r_{k}^{\min }$ & $r_{k}^{\max }$ & $r_{k}^{1 \text { min }}$ & $r_{k}^{1 \max }$ & $r_{k}^{2 \min }$ & $r_{k}^{2 \max }$ \\
\hline PAB & 1 & 4 & 5 & 1 & 14 & 3 & 5 \\
SPDB & 2 & 2 & 2 & 2 & 5 & 2 & 4 \\
HXB & 3 & 13 & 13 & 7 & 16 & 7 & 13 \\
CMBC & 4 & 1 & 2 & 10 & 15 & 1 & 8 \\
CMB & 5 & 1 & 6 & 2 & 12 & 1 & 12 \\
IB & 6 & 4 & 4 & 6 & 15 & 1 & 4 \\
ABC & 7 & 13 & 16 & 6 & 12 & 15 & 16 \\
BOCOM & 8 & 9 & 10 & 1 & 16 & 6 & 11 \\
ICBC & 9 & 9 & 13 & 1 & 14 & 11 & 15 \\
CEB & 10 & 6 & 6 & 3 & 13 & 3 & 7 \\
CCB & 11 & 7 & 12 & 5 & 12 & 9 & 14 \\
BOC & 12 & 6 & 8 & 7 & 15 & 6 & 16 \\
CNCB & 13 & 5 & 6 & 1 & 8 & 7 & 12 \\
BJB & 14 & 6 & 7 & 1 & 5 & 5 & 14 \\
NJB & 15 & 13 & 15 & 6 & 11 & 7 & 15 \\
NBB & 16 & 9 & 9 & 11 & 13 & 6 & 9 \\
\hline
\end{tabular}

\section{Conclusions and direction for future research}

In previous DEA literature, each DMU is evaluated by using the most favorable weights. However, it ignores other feasible weights. To overcome this problem, Salo and Punkka (2011) deemed each DMU as a "Black Box", and developed mix-integer models to obtain the ranking intervals over sets of all feasible weights. In this paper, we expand their method by considering the internal structure of the DMUs. We extend their method to compute ranking intervals for a two-stage production system and illustrate the method by revisiting reported DEA studies. Thus the "Black Box" is opened, and more accurate information on the ranking intervals for the overall system and both subsystems is provided by to the decision maker. 
The efficiency measure used in this paper is radial, some non-radial measures have also been proposed in the literature, such as the slack-based measure (Tone and Tsutsui, 2009; Tone and Tsutsui, 2010). Obtaining the ranking intervals for two-stage production systems based on non-radial DEA is another interesting avenue to explore in the future.

\section{Acknowledgements}

The authors would like to thank three anonymous reviewers and the editor of Journal of Operational research Society for their insightful comments and suggestions, which as results this paper has been improved substantially. The authors would also like to express their thanks to Professor Alec Morton for his valuable suggestions on this paper. This research was supported by Funds of National Natural Science Foundation of China (71701111, 71271196, 71671172 and 71225002, 71701102), Funds for Creative

Research Groups of the National Natural Science Foundation of China and University of Science and Technology of China (71121061 and WK2040160008), the Fund for International Cooperation and Exchange of the National Natural Science Foundation of China (71110107024).

\section{References:}

Alcaraz, J., Ramón, N., Ruiz, J.L., \& Sirvent, I. (2013). Ranking ranges in cross-efficiency evaluations. European journal of operational research, 226, 516-521.

Amirteimoori, A., \& Kordrostami, S. (2005). Allocating fixed costs and target setting: a DEA-based approach. Applied Mathematics and Computation, 171, 136-151.

Asmild M., Paradi J. C., \& Pastor J. T.(2012). DEA based models for reallocations of police personnel. OR spectrum, 34, 921-941.

Avkiran, N. K., \& McCrystal, A. (2012). Sensitivity analysis of network DEA: NSBM versus NRAM. Applied Mathematics and Computation, 218, 11226-11239.

Banker R.D., Chang H., Zheng Z.Q. (2017). On the use of super-efficiency procedures for ranking efficient units and identifying outliers. Annals of Operations Research, 250 (1): 21-35.

Basso A., \& Funari S.( 2001). A data envelopment analysis approach to measure the mutual fund performance. European Journal of Operational Research, 135, 477-492.

Berger A.N., Humphrey D.B. (1997), Efficiency of financial institutions: International survey and directions for future research, European Journal of Operational Research, 98,175-212.

Bi, G.-B., Song, W., Zhou, P., \& Liang, L. (2014). Does environmental regulation affect energy efficiency in China's thermal power generation? Empirical evidence from a slacks-based DEA model. Energy Policy, 66, 537-546. 
Charnes, A., Cooper, W.W., \& Rhodes, E. (1978). Measuring the efficiency of decision making units. European journal of operational research, 2, 429-444.

Chen X., Skully M., Brown K. (2005). Banking efficiency in China: Application of DEA to pre- and post-deregulation eras: 1993-2000. China Economic Review,16 (3), 229-245.

Chen Y (2005). Measuring super-efficiency in DEA in the presence of infeasibility. European Journal of Operational Research,161(2), 545-551.

Chen Z., \& Lin R. (2006). Mutual fund performance evaluation using data envelopment analysis with new risk measures. OR Spectrum, 28, 375-398.

Chen, C.-M., \& Zhu, J. (2011). Efficient resource allocation via efficiency bootstraps: an application to R\&D project budgeting. Operations Research, 59, 729-741.

Chen, Y., \& Zhu, J. (2004). Measuring information technology's indirect impact on firm performance. Information Technology and Management, 5, 9-22.

Chen, Y., Cook, W. D., Li, N., \& Zhu, J. (2009a). Additive efficiency decomposition in two-stage DEA. European journal of operational research, 196, 1170-1176.

Chen, Y., Liang, L., \& Zhu, J. (2009b). Equivalence in two-stage DEA approaches. European journal of operational research, 193, 600-604.

Cummins, J. D., \& Xie, X. (2008). Mergers and acquisitions in the US property-liability insurance industry: Productivity and efficiency effects. Journal of Banking \& Finance, 32, 30-55.

Drake L., Hall M.J.B., Simper R. (2006). The impact of macroeconomic and regulatory factors on bank efficiency: A non-parametric analysis of Hong Kong's banking system, Journal of Banking and Finance, 30, 1443-1466.

Du J, Wang J, Chen Y, Chou SY, Zhu J. (2014). Incorporating health outcomes in Pennsylvania hospital efficiency: an additive super-efficiency DEA approach. Annals of Operations Research, $221(1), 161-172$.

Dyson, R. G., Allen, R., Camanho, A. S., Podinovski, V. V., Sarrico, C. S., \& Shale, E. A. (2001). Pitfalls and protocols in DEA. European journal of operational research, 132, 245-259.

Eling M., Schaper P. (2017). Under pressure: how the business environment affects productivity and efficiency of European life insurance companies. European Journal of Operational Research, 258(3),1082-1094.

Emrouznejad, A., Yang G., (2018). A survey and analysis of the first 40 years of scholarly literature in DEA: 1978-2016. Socio-Economic Planning Sciences, 61, 4-8.

Fukuyama, H., \& Weber, W. L. (2009). A directional slacks-based measure of technical inefficiency. Socio Economic Planning Sciences, 43, 274-287.

Fukuyama, H., \& Weber, W. L. (2010). A slacks-based inefficiency measure for a two-stage system with bad outputs. Omega, 38, 398-409.

Halkos, G. E., \& Tzeremes, N. G. (2013). Estimating the degree of operating efficiency gains from a potential bank merger and acquisition: a DEA bootstrapped approach. Journal of Banking \& Finance, 37, 1658-1668.

Huang C.W., Ho F.N., Chiu Y.H. (2014). Measurement of tourist hotels' productive efficiency, occupancy, and catering service effectiveness using a modified two-stage DEA model in Taiwan. Omega, 48, 49-59.

Kao C. (2014). Efficiency decomposition for general multi-stage systems in data envelopment analysis. European Journal of Operational Research, 232(1), 117-124.

Kao C., Hwang S.N. (2008). Efficiency decomposition in two-stage data envelopment analysis: An 
application to non-life insurance companies in Taiwan. European Journal of Operational Research, 185, 418-429.

Kao, C., \& Hwang, S.-N. (2008). Efficiency decomposition in two-stage data envelopment analysis: An application to non-life insurance companies in Taiwan. European journal of operational research, 185, 418-429.

Kao, C., \& Hwang, S.-N. (2010). Efficiency measurement for network systems: IT impact on firm performance. Decision Support Systems, 48, 437-446.

Lozano S., Gutiérrez E., Moreno P. (2013). Network DEA approach to airports performance assessment considering undesirable outputs. Applied Mathematical Modelling, 37(4), 16651676.

Maudos J., Pastor J.M., Perez F. (2002), Competition and efficiency in the Spanish banking sector: the importante of specialization, Applied Financial Economics, 12, 505-516.

Paradi, J.C., Rouatt, S., \& Zhu, H. Y. (2011). Two-stage evaluation of bank branch efficiency using data envelopment analysis. Omega, 39, 99-109.

Pasiouras F., (2008a), International evidence on the impact of regulations and supervision on banks' technical efficiency: an application of two-stage data envelopment analysis, Review of Quantitative Finance and Accounting, 30, 187-223.

Ray SC (2008). The directional distance function and measurement of super-efficiency: an application to airlines data. Journal of the Operational Research Society, 59(6), 788-797.

Sadjadia S.J., Omrani H., Abdollahzadeh S., Alinaghian M. \& Mohammadi H. (2011). A robust super-efficiency data envelopment analysis model for ranking of provincial gas companies in Iran. Expert Systems with Applications 38(9), 10875-10881.

Salo, A., \& Punkka, A. (2011). Ranking intervals and dominance relations for ratio-based efficiency analysis. Management Science, 57, 200-214.

Seiford, L. M., \& Zhu, J. (1999). Profitability and marketability of the top 55 US commercial banks. Management Science, 45, 1270-1288.

Sexton T.R., Lewis H.F.(2003). Two-stage DEA: An application to major league baseball. Journal of Productivity Analysis, 19,227-249.

Shi X., Li Y.J., Emrouznejad A., Xie J.H. \& Liang L. (2017). Estimation of potential gains from bank mergers: A novel two-stage cost efficiency DEA model. Journal of the Operational Research Society, 68,1045-1055

Tsui W.H., Balli H.O., Gilbey A., Gow H. (2014). Operational efficiency of Asia-Pacific airports. Journal of Air Transport Management, 40,16-24.

Wang K., Huang W., Wu J., Liu Y.N. (2014). Efficiency measures of the Chinese commercial banking system using an additive two-stage DEA. Omega, 44, 5-20.

Wu H.Q., Lv K., Liang L. \& Hu H.H. (2017). Measuring performance of sustainable manufacturing with recyclable wastes: A case from China's iron and steel industry. Omega, 66, 38-47.

Yang, F., Ang, S., Xia, Q., \& Yang, C. (2012). Ranking DMUs by using interval DEA cross efficiency matrix with acceptability analysis. European journal of operational research, 223, 483-488.

Zha, Y., \& Liang, L. (2010). Two-stage cooperation model with input freely distributed among the stages. European journal of operational research, 205, 332-338.

Zhao L.S., Sun C.Z., Liu F.C. (2017). Interprovincial two-stage water resource utilization efficiency under environmental constraint and spatial spillover effects in China. Journal of Cleaner 
Production, 164,715-725.

Zhou, P., Poh, K. L., \& Ang, B. W. (2007). A non-radial DEA approach to measuring environmental performance. European journal of operational research, 178, 1-9.

Zhu J. (2001). Super-efficiency and DEA sensitivity analysis. European Journal of Operational Research, 129 (2), 443-455. 


\section{Appendix}

\section{Proof of Proposition 1}

Proof. The constraints (7.1) and (7.2) are included in model (7) in order to identify DMUs with larger efficiency scores for subsystem 1 and subsystem 2 than that of $D M U_{k}$. The constraint (7.3) $\sum_{r} u_{r} y_{r k}=\sum_{d} w_{d} z_{d k}=1$ implies $D M U_{k}$ 's efficiency score in subsystem 2 is one, namely $E_{k}^{2}=1$; and the constraint (7.4) $\sum_{d} w_{d} z_{d k}=\sum_{i} v_{i} x_{i k}=1$ implies $D M U_{k}$, efficiency score in subsystem 1 is one, namely $E_{k}^{1}=1$. Hence, for every feasible choice of DEA weights, if $E_{l}^{2}>E_{k}^{2}$ holds, that is $\frac{E_{l}^{2}}{E_{k}^{2}}=\frac{\sum_{r} u_{r} y_{r l} / \sum_{d} w_{d} z_{d l}}{\sum_{r} u_{r} y_{r k} / \sum_{d} w_{d} z_{d k}}>1$ (or $\left.\sum_{r} u_{r} y_{r l}>\sum_{d} w_{d} z_{d l}\right)$, then $p_{l}^{2}$ will be necessarily 1 . In the same manner, if $E_{l}^{1}>E_{k}^{1}\left(\right.$ or $\left.\sum_{d} w_{d} z_{d l}>\sum_{i} v_{i} x_{i l}\right)$ holds, then $p_{l}^{1}$ will be necessarily 1 .

While $\quad$ if $\quad E_{l}^{2} \leq E_{k}^{2} \quad$ and $\quad E_{l}^{1} \leq E_{k}^{1} \quad$ (or $\quad \sum_{r} u_{r} y_{r l} \leq \sum_{d} w_{d} z_{d l} \quad$ and $\left.\sum_{d} w_{d} z_{d l} \leq \sum_{i} v_{i} x_{i l}\right)$ hold, then $p_{l}^{1}$ and $p_{l}^{2}$ can be either 0 or 1 . As we minimize the sum of $p_{l}^{1}+p_{l}^{2}$ in the objective (7). Thus, at optimum, $E_{l}^{2}>E_{k}^{2}$ and $E_{l}^{1}>E_{k}^{1}$ are necessarily associated with $p_{l}^{1 *}=1$ and $p_{l}^{2 *}=1$, while $E_{l}^{2} \leq E_{k}^{2}$ and $E_{l}^{1} \leq E_{k}^{1}$ are necessarily associated with $p_{l}^{1 *}=0$ and $p_{l}^{2 *}=0$. Consequently, the objective ofmodel (7) identifies the DMUs with strictly larger efficiency scores for both two subsystems than that of $D M U_{k}$.

\section{Proof of Proposition 2}

Proof. The proof is similar to Proposition 1.

For every feasible choice of DEA weights, if $E_{l}^{2} \geq E_{k}^{2}$ (or $\sum_{r} u_{r} y_{r l} \geq \sum_{d} w_{d} z_{d l}$ ) holds, then $p_{l}^{2}$ will be necessarily 0 or 1 . In the same manner, if $E_{l}^{1} \geq E_{k}^{1}$ (or $\left.\sum_{d} w_{d} z_{d l} \geq \sum_{i} v_{i} x_{i l}\right)$ holds, then $p_{l}^{1}$ will be either 0 or 1 .

While if $E_{l}^{2}<E_{k}^{2}$ and $E_{l}^{1}<E_{k}^{1}$ hold, then $p_{l}^{1}$ and $p_{l}^{2}$ can be necessarily 0 . 
As we maximize the sum of $p_{l}^{1} * p_{l}^{2}$ in the objective (10), at optimum, $E_{l}^{2} \geq E_{k}^{2}$ and $E_{l}^{1} \geq E_{k}^{1}$ are associated with $p_{l}^{1}=1$ and $p_{l}^{2}=1$, while $E_{l}^{2}<E_{k}^{2}$ and $E_{l}^{1}<E_{k}^{1}$ are associated with $p_{l}^{1}=0$ and $p_{l}^{2}=0$. Consequently, the objective $r_{k}^{\max }$ of model (10) accounts for the maximum number of DMUs with efficiency scores larger than or equal to those of $D M U_{k}$ (excluding $D M U_{k}$ ). Thus, the objective inmodel (10) obtains the worst ranking of $D M U_{k}$ which has a two-stage production system.

\section{Proof of Proposition 3}

Proof. The constraint (11.3) $\sum_{r} u_{r} y_{r k}=\sum_{d} w_{d} z_{d k}=1$ and (11.4) $\sum_{d} w_{d} z_{d k}=\sum_{i} v_{i} x_{i k}=1$ imply $D M U_{\mathrm{k}}$ 's efficiency scores in subsystem 2 and subsystem 1are 1, respectively. That is, $E_{k}^{2}=1$ and $E_{k}^{1}=1$. For every feasible choice of weights, if $E_{l}^{1}>E_{k}^{1}$ (or $\sum_{d} w_{d} z_{d l}>\sum_{i} v_{i} x_{i l}$ ), then $p_{l}^{1}$ will be necessarily 1 . Similarly, if $E_{l}^{2}>E_{k}^{2}$ (or $\left.\sum_{r} u_{r} y_{r l}>\sum_{d} w_{d} z_{d l}\right)$, then $p_{l}^{2}$ will be necessarily 1 .

While if $E_{l}^{1} \leq E_{k}^{1}$, then $p_{l}^{1}$ can be either 0 or 1 . Similarly, if $E_{l}^{2} \leq E_{k}^{2}$, then $p_{l}^{2}$ can be either 0 or 1 . As we minimize the sum of $p_{l}^{1}$ in the objective (11), at optimum, $E_{l}^{1}>E_{k}^{1}$ is necessarily associated with $p_{l}^{1}=1$, while $E_{l}^{1} \leq E_{k}^{1}$ isnecessarily associated with $p_{l}^{1}=0$. Consequently, the objective ofmodel (11) accounts for the minimum number of DMUs with efficiency scores larger than that of $D M U_{k}$ (excluding $D M U_{k}$ ). Thus, the objective in model (11) obtains the best ranking of $D M U_{k}$ in subsystem 1.

\section{Proof of Proposition4}

Proof. For every feasible choice of DEA weights of each DMU, if $E_{l}^{1} \geq E_{k}^{1}$ (or $\sum_{d} w_{d} z_{d l} \geq \sum_{i} v_{i} x_{i l}$ ), then $p_{l}^{1}$ will be necessarily 0 or 1 . While if $E_{l}^{1}<E_{k}^{1}$ (or 
$\left.\sum_{d} w_{d} z_{d l}<\sum_{i} v_{i} x_{i l}\right)$, then $p_{l}^{1}$ can be necessarily 0 . As we maximize the sum of $p_{l}^{1}$ in the objective (12), at optimum $E_{l}^{1} \geq E_{k}^{1}$ is necessarily associated with $p_{l}^{1}=1$, while $E_{l}^{1}<E_{k}^{1}$ is necessarily associated with $p_{l}^{1}=0$.

Consequently, the objective $r_{k}^{1 \max }$ of model (12) accounts for the maximum number of DMUs with efficiency scores larger than or equal to that of $D M U_{k}$ (excluding $D M U_{k}$ ).Thus, the objective in model (12) obtains the worst ranking of $D M U_{k}$ in subsystem 1 . 